\title{
Adaptive High-Order Finite Difference Analysis of 2D Quenching- Type Convection-Reaction-Diffusion Equation
}

\author{
Xiaoliang Zhu $\mathbb{D}^{1,2}$ and Yongbin Ge $\mathbb{D}^{1}$ \\ ${ }^{1}$ Institute of Applied Mathematics and Mechanics, Ningxia University, Yinchuan 750021, China \\ ${ }^{2}$ School of Computer and Engineering, North Minzu University, Yinchuan 750021, China \\ Correspondence should be addressed to Yongbin Ge; gyb@nxu.edu.cn
}

Received 2 May 2020; Revised 19 July 2020; Accepted 4 August 2020; Published 29 October 2020

Academic Editor: Sergey Shmarev

Copyright (c) 2020 Xiaoliang Zhu and Yongbin Ge. This is an open access article distributed under the Creative Commons Attribution License, which permits unrestricted use, distribution, and reproduction in any medium, provided the original work is properly cited.

\begin{abstract}
Quenching characteristics based on the two-dimensional (2D) nonlinear unsteady convection-reaction-diffusion equation are creatively researched. The study develops a $2 \mathrm{D}$ compact finite difference scheme constructed by using the first and the second central difference operator to approximate the first-order and the second-order spatial derivative, Taylor series expansion rule, and the reminder-correction method to approximate the three-order and the four-order spatial derivative, respectively, and the forward difference scheme to discretize temporal derivative, which brings the accuracy resulted meanwhile. Influences of degenerate parameter, convection parameter, and the length of the rectangle definition domain on quenching behaviors and performances of special quenching cases are discussed and evaluated by using the proposed scheme on the adaptive grid. It is feasible for the paper to offer potential support for further research on quenching problem.
\end{abstract}

\section{Introduction}

As a common class of thermodynamic problems, quenching phenomena research has been widely applied in engineering area. There exist some typical researches including the flow, thermal properties, and its working mechanism of an object in heat associated with quenching phenomena $[1,2]$, the forced convection quench of hot sheets in super-cooled liquid [3], the quench process speed-up, and its quench characteristics in porous interface processing $[4,5]$. In recent years, more and more scholars concern quenching problem built on parabolic equations [6-10], in which the authors depended on the different parabolic equations to analyze corresponding quench features. Additionally, quenching phenomena based on parabolic equation systems have attracted researchers' attentions [11-13]. Nonlinear degeneration singularity reaction-diffusion equations or convectionreaction-diffusion equations, as a branch of parabolic equations, have been usually employed to handle quenching problems. References [14-16] focused on the quench- ing phenomena of the nonlinear degeneration singularity reaction-diffusion equations whereas Refs. $[17,18]$ focused on the quenching phenomena of the nonlinear degeneration singularity convection-reaction-diffusion equations. Selcuk discussed quench performances of solution and its time derivative and estimation of quenching time under special conditions [14]. Ge et al. devoted to an adaptive compact difference scheme to solve quench problems [15]. Beauregard analyzed quenching properties of the fractional Kawarada equation by using a new numerical method and proved the proposed method monotonic, nonnegative, and linearly stable [16]. Zhou et al. theoretically investigated quenching characteristics of the nonlinear degeneration singularity convection-reaction-diffusion equation [17]. Zhu and Rui constructed a high-accuracy method with adaptation mechanism for studying quenching behaviors and analyzed the influence of important elements on quenching [18].

References [6-18] concentrated on the quenching phenomena of the $1 \mathrm{D}$ heat equations. The quenching phenomena of the $2 \mathrm{D}$ heat equations are described as follows. 
References [19-21] used numerical method with adaptive algorithm to research degeneration singularity problem of the two-dimensional nonlinear reaction-diffusion equations. A Peaceman-Rachford finite difference scheme based on semiadaptive grids with stability and convergence under certain condition was posed to analyze the quenching behaviors of the $2 \mathrm{D}$ reaction-diffusion equation in Ref. [19]. Numerical analyses showed that the method is very efficient and reliable. An adaptive Peaceman-Rachford-Strang splitting method on exponentially evolving meshes was constructed to resolve the 2D problem of quenching type in Ref. [20]. Computational experiments stated that the method is of the satisfactory effectiveness, efficiency, and numerical stability. An exponential splitting method with spatial semidiscretization on arbitrary nonuniform meshes was investigated to explore the 2D degenerate stochastic Kawarada equation in Ref. [21]. Zhu and Ge developed an adaptive ADI strategy to simulate quenching phenomena based on 2D convection-reaction-diffusion equation [22]. Numerical cases illustrated that the method is of the positivity, monotonicity, and numerical stability. The low-accuracy strategies were adopted in the most aforementioned researches [6-15, 19-21]. There are only Refs. [15, 18, 22] delivering the high-accuracy strategies, in which the first two refer to the one-dimensional problem and the last one refers to the two-dimensional problem. It is well known that the development of high-order finite difference scheme of the 2D nonlinear convection-reaction-diffusion equations is maturing [23-25]. Reference [23] developed a new highorder difference approach for the $2 \mathrm{D}$ convection-reactiondiffusion equation with a small diffusivity, which firstly posed the 1D high-accuracy difference scheme and then extended it to the $2 \mathrm{D}$ case a nine-point stencil by using alternating direction algorithm and last resolve the 2D steady incompressible Navier-Stokes equations. In order to simulate groundwater pollution problems, Li et al. put forward a fourth-order compact difference scheme with unconditional stability and the second-order temporal accuracy, the fourth-order spatial accuracy built on the $2 \mathrm{D}$ convection-reaction-diffusion model, which was efficient through experiments [24]. Wu and Zhai combined exponential transformation and quadratic interpolation polynomial with Padé approximation to study the 2D time-fractional convection-dominated diffusion equation, which owned the higher accuracy and used alternating direction implicit algorithm to alleviate computational amount [25].

In summary, it is easily found that there are much more low-order strategies than high-order strategies to solve degenerate singularity problems of the $2 \mathrm{D}$ reactiondiffusion equation. Especially, high-order difference schemes received few attentions for solving quenching problem of the two-dimensional convection-reaction-diffusion equation. It is the high-precision algorithms that have advantages in dealing with such problems because of its high accuracy and efficiency. So it is a meaningful try to explore the high-order compact difference schemes on adaptation mesh for analyzing quenching problems of the $2 \mathrm{D}$ degenerate singular reaction-diffusion equation with convection function. Going down this idea, we extend this study from Ref. [18] and construct a compact difference scheme on adaptive grid for solving the $2 \mathrm{D}$ unsteady convection-reaction-diffusion equation to explain the corresponding quenching phenomena. According to Ref. [18], we extend its 1D high-order compact finite difference scheme on adaptive mesh to a $2 \mathrm{D}$ strategy and use it to analyze the quenching behaviors of the $2 \mathrm{D}$ convection-reaction-diffusion combustion model. There exist three contributions of this paper. Firstly, we represent a new 2D high-order compact difference scheme for solving the corresponding unsteady convection-reaction-diffusion equation and give its accuracy performances. Secondly, we apply the scheme to explain the $2 \mathrm{D}$ reaction-diffusion of quenching type with and without convection function, respectively. Thirdly, we investigate a series of quenching characteristics including quenching time, quenching location, and so on, from which we can discover impacts of the parameters $q, b$, and $\alpha(\beta)$ on quenching behaviors. There are five parts in the paper. Section 1 introduces the theme of this study. Section 2 describes carefully the proposed scheme of high-order accuracy. Section 3 introduces adaptive mesh algorithm. Section 4 stimulates some typical numerical samples to explore and explain quenching problems. Section 5 draws the conclusion.

\section{2D Problem and Difference Scheme}

2.1. 2D Nonlinear Convection-Reaction-Diffusion Equation. The typical convection-reaction-diffusion equation of quenching type is written as

$$
\begin{aligned}
\sigma(x, y) u_{t}= & u_{x x}+u_{y y}+c_{1}(x, y) u_{x}+c_{2}(x, y) u_{y} \\
& +f(u),(x, y) \in \Omega, t \in(0, T) .
\end{aligned}
$$

Its boundary conditions are

$$
u(0, y, t)=u(\alpha, y, t)=u(x, 0, t)=u(x, \beta, t)=0, t \in(0, T) .
$$

Its initial conditions is

$$
\left.u(x, y, 0)=u_{0}(x, y),(x, y) \in 0, \alpha\right) \times(0, \beta)
$$

This semilinear degenerate problem model involving two spatial dimensions is regarded as Eq. (1) with the boundary and initial conditions of Eqs. (2) and (3). The solution $u(x, y$ , $t)$ represents the temperature of the combustion chamber. $\Omega \in(0, \alpha) \times(0, \beta)$ refers to the smooth domain of the rectangle combustor in which $\alpha>0, \beta>0$, and combustion chamber sizes $\alpha$ and $\beta$ are the length of the definition area, $\partial \Omega$, and is its boundary. $c_{1}(x, y)$ and $c_{2}(x, y)$ are the convection functions of $x$ and $y$, and $\sigma(x, y)=\left(x^{2}+y^{2}\right)^{q / 2}$ is the degeneracy function and degeneration parameter $q \geq 0$. The singularity source $f($ $u)=1 /(1-u)^{\theta}$ is strictly increasing for $0 \leq u_{0}<1$ with $f(0)=f_{0}>0, \lim _{u \rightarrow 1} f(u)=\infty$, and singularity parameter $\theta$ is the power of the singular source term $1 /(1-u)^{\theta}$.

With the aid of the intermediate variables $\hat{x}$ and $\hat{y}$, we replace $\hat{x}=x / \alpha$ and $\hat{y}=y / \beta$ into Eq. (1) which can be defined as 


$$
\begin{aligned}
\sigma(\alpha \widehat{x}, \beta \widehat{y}) u_{t}= & \frac{1}{\alpha^{2}} u_{\widehat{x} \widehat{x}}+\frac{1}{\beta^{2}} u_{\widehat{y} \widehat{y}}+\frac{c_{1}(\alpha \widehat{x}, \beta \widehat{y})}{\alpha} u_{\widehat{x}}+\frac{c_{2}(\alpha \widehat{x}, \beta \widehat{y})}{\beta} u_{\widehat{y}} \\
& +f(u),(\alpha \widehat{x}, \beta \widehat{y}) \in \Omega, t \in(0, T) .
\end{aligned}
$$

For the convenience of expression, we use $x$ and $y$ instead of $\hat{x}$ and $\hat{y}$. Then, the above formulation can be rearranged as

$$
\begin{aligned}
\sigma(x, y) u_{t}= & \frac{1}{\alpha^{2}} u_{x x}+\frac{1}{\beta^{2}} u_{y y}+\frac{c_{1}(x, y)}{\alpha} u_{x}+\frac{c_{2}(x, y)}{\beta} u_{y} \\
& +f(u),(x, y) \in \Omega, t \in(0, T)
\end{aligned}
$$

where $\sigma(x, y)=\left(\alpha^{2} x^{2}+\beta^{2} y^{2}\right)^{q / 2}, \Omega \in(0,1) \times(0,1)$. Correspondingly, the boundary conditions of Eq. (5) are

$$
u(0, y, t)=u(1, y, t)=u(x, 0, t)=u(x, 1, t)=0, t \in(0, T) .
$$

The initial conditions of Eq. (5) is

$$
u(x, y, 0)=u_{0}(x, y),(x, y) \in(0,1) \times(0,1) .
$$

In the physical application, we rely on Eqs. (5)-(7) to compute $u$ and $u_{t}$. Through observing a large number of values of $u$ and $u_{t}$, we can capture quenching moment, i.e., quenching occurs when $u$ infinitely close to 1 and $u_{t}$ becomes so huge that its value blows up.

2.2. The Proposed Compact Difference Scheme. According to the idea given in [18], we employ the first and the second central difference operator to approximate the first-order and the second-order spatial derivatives of $x$ - direction and $y-$ direction, respectively, and the forward difference operator to discrete temporal derivative. The proposed high-accuracy finite difference scheme of Eq. (5) is deduced below. After the first-order and the second-order spatial derivatives of $x$ - direction and $y$-direction are discretized on the nonuniform mesh, respectively, a scheme approximating the $2 \mathrm{D}$ unsteady convection-diffusion equation dispersed at point ( $\left.x_{k}, y_{j}\right)$ can be written as

$$
\begin{aligned}
& \left\{\frac{2-\alpha\left(x_{R}-x_{L}\right) c_{1}}{2 \alpha^{2} \sigma} \delta_{x}^{2}+\frac{c_{1}}{\alpha \sigma} \delta_{x}+\frac{2-\beta\left(y_{R}-y_{L}\right) c_{2}}{2 \beta^{2} \sigma} \delta_{y}^{2}\right. \\
& \left.\quad+\frac{c_{2}}{\beta \sigma} \delta_{y}\right\} u_{k, j}+\Psi_{1 x}\left(u_{x x x}\right)_{k, j}+\Psi_{2 x}\left(u_{x x x x}\right)_{k, j}+\Psi_{3 x}\left(u_{x x x x x}\right)_{k, j} \\
& \quad+\Psi_{1 y}\left(u_{y y y}\right)_{k, j}+\Psi_{2 y}\left(u_{y y y y}\right)_{k, j}+\Psi_{3 y}\left(u_{y y y y y}\right)_{k, j} \\
& =\left\{u_{t}-\frac{f}{\sigma}(u)\right\}_{k, j, l}+O\left(\frac{x_{R}^{5}+x_{L}^{5}}{x_{R}+x_{L}}\right)+O\left(\frac{y_{R}^{5}+y_{L}^{5}}{y_{R}+y_{L}}\right),
\end{aligned}
$$

where

$$
\begin{aligned}
& \Psi_{1 x}=-\frac{2\left(x_{R}-x_{L}\right)+\alpha x_{R} x_{L} c_{1}}{6 \alpha^{2} \sigma}, \\
& \Psi_{2 x}=-\frac{2\left(x_{R}^{2}-x_{R} x_{L}+x_{L}^{2}\right)+\alpha\left(x_{R}-x_{L}\right) x_{R} x_{L} c_{1}}{24 \alpha^{2} \sigma}, \\
& \Psi_{3 x}=-\frac{2\left(x_{R}-x_{L}\right)\left(x_{R}^{2}+x_{L}^{2}\right)+\alpha\left(x_{R}^{3} x_{L}-x_{R}^{2} x_{L}^{2}+x_{L}^{3} x_{R}\right) c_{1}}{120 \alpha^{2} \sigma}, \\
& \Psi_{1 y}=-\frac{2\left(y_{R}-y_{L}\right)+\beta y_{R} y_{L} c_{2}}{6 \beta^{2} \sigma}, \\
& \Psi_{2 y}=-\frac{2\left(y_{R}^{2}-y_{R} y_{L}+y_{L}^{2}\right)+\beta\left(y_{R}-y_{L}\right) y_{R} y_{L} c_{2}}{24 \beta^{2} \sigma}, \\
& \Psi_{3 y}=-\frac{2\left(y_{R}-y_{L}\right)\left(y_{R}^{2}+y_{L}^{2}\right)+\beta\left(y_{R}^{3} y_{L}-y_{R}^{2} y_{L}^{2}+y_{L}^{3} y_{R}\right) c_{2}}{120 \beta^{2} \sigma} .
\end{aligned}
$$

We use Taylor series to obtain the derivative expansions of $x$-direction: $\left(u_{x x x}\right)_{k, j}$ and $\left(u_{x x x x}\right)_{k, j}$, and the derivative expansions of $y$-direction: $\left(u_{y y y}\right)_{k, j}$ and $\left(u_{y y y y}\right)_{k, j}$ from Eq. (5). The four expressions are substituted into the corresponding terms in Eq. (8). $O(\Delta)=O\left(x_{R}^{5}+x_{L}^{5} / x_{R}+x_{L}\right)+O($ $\left.y_{R}^{5}+y_{L}^{5} / y_{R}+y_{L}\right)-\Psi_{2 y}\left(u_{y y y y}\right)_{k, j}-\Psi_{3 y}\left(u_{y y y y y}\right)_{k, j}$, which is the truncation error of Eq. (8). Omitting the truncation errors, we consider the situation of point $(k, j, n)$ for Eq. (8)

$$
\begin{aligned}
& \left\{\kappa_{1 x} \delta_{x}+\kappa_{2 x} \delta_{x}^{2}+\kappa_{1 y} \delta_{y}+\kappa_{2 y} \delta_{y}^{2}\right\} u_{k, j}^{n}+\left\{\kappa_{3} u_{t}+\kappa_{4 x} u_{t x}+\kappa_{5 x} u_{t x x}\right. \\
& \left.\quad+\kappa_{4 y} u_{t y}+\kappa_{5 y} u_{t y y}+\kappa_{6} u_{x y}+\kappa_{7 x} u_{x x y}+\kappa_{7 y} u_{x y y}+\kappa_{8} u_{x x y y}\right\}_{k, j}^{n} \\
& =\left\{-\frac{1}{\sigma}+\kappa_{9 x} \delta_{x}+\kappa_{10 x} \delta_{x}^{2}+\kappa_{9 y} \delta_{y}+\kappa_{10 y} \delta_{y}^{2}\right\} f_{k, j}^{n}+O(\Delta),
\end{aligned}
$$

where

$$
\begin{aligned}
\kappa_{1 x}= & \frac{c_{1}}{\alpha \sigma}-\alpha\left(\Psi_{1 x}-\alpha \Psi_{2 x} c_{1}\right)\left(c_{1}\right)_{x}-\alpha \Psi_{2 x}\left(c_{1}\right)_{x x} \\
& -\frac{\beta^{2}}{\alpha}\left(\Psi_{1 y}-\beta \Psi_{2 y} c_{2}\right)\left(c_{1}\right)_{y}-\frac{\beta^{2} \Psi_{2 y}}{\alpha}\left(c_{1}\right)_{y y}, \\
\kappa_{2 x}= & \frac{2-\alpha\left(x_{R}-x_{L}\right) c_{1}}{2 \alpha^{2} \sigma}-\alpha\left(\Psi_{1 x}-\alpha \Psi_{2 x} c_{1}\right) c_{1}-2 \alpha \Psi_{2 x}\left(c_{1}\right)_{x}, \\
\kappa_{1 y}= & \frac{c_{2}}{\beta \sigma}-\beta\left(\Psi_{1 y}-\beta \Psi_{2 y} c_{2}\right)\left(c_{2}\right)_{y}-\beta \Psi_{2 y}\left(c_{2}\right)_{y y} \\
& -\frac{\alpha^{2}}{\beta}\left(\Psi_{1 x}-\alpha \Psi_{2 x} c_{1}\right)\left(c_{2}\right)_{x}-\frac{\alpha^{2} \Psi_{2 x}}{\beta}\left(c_{2}\right)_{x x}, \\
\kappa_{2 y}= & \frac{2-\beta\left(y_{R}-y_{L}\right) c_{2}}{2 \beta^{2} \sigma}-\beta\left(\Psi_{1 y}-\beta \Psi_{2 y} c_{2}\right) c_{2}-2 \beta \Psi_{2 y}\left(c_{2}\right)_{y},
\end{aligned}
$$




$$
\begin{array}{crl}
\kappa_{3}=\alpha^{2}\left(\Psi_{1 x}-\alpha \Psi_{2 x} c_{1}\right) \sigma_{x}+\alpha^{2} \Psi_{2 x} \sigma_{x x} & \text { Provided } O(\Delta)=O\left(\Delta+\tau^{2}\right), \text { Eq. (14) can be derived as } \\
+\beta^{2}\left(\Psi_{1 y}-\beta \Psi_{2 y} c_{2}\right) \sigma_{y}+\beta^{2} \Psi_{2 y} \sigma_{y y}-1, & \left\{\kappa_{1 x} \delta_{x}+\kappa_{2 x} \delta_{x}^{2}+\kappa_{1 y} \delta_{y}+\kappa_{2 y} \delta_{y}^{2}\right\} u_{k, j}^{n}+\kappa_{3} \delta_{t}^{+} u_{k, j}^{n} \\
\kappa_{4 x}=\alpha^{2}\left(\Psi_{1 x}-\alpha \Psi_{2 x} c_{1}\right) \sigma+2 \alpha^{2} \Psi_{2 x} \sigma_{x}, \kappa_{4 y} & +\left\{\left(\kappa_{4 x}-\frac{\kappa_{3} \tau_{n} c_{1}}{2 \alpha \sigma}\right) u_{t x}+\left(\kappa_{5 x}-\frac{\kappa_{3} \tau_{n}}{2 \alpha^{2} \sigma}\right) u_{t x x}\right. \\
=\beta^{2}\left(\Psi_{1 y}-\beta \Psi_{2 y} c_{2}\right) \sigma+2 \beta^{2} \Psi_{2 y} \sigma_{y}, & \left.+\left(\kappa_{4 y}-\frac{\kappa_{3} \tau_{n} c_{2}}{2 \beta \sigma}\right) u_{t y}+\left(\kappa_{5 y}-\frac{\kappa_{3} \tau_{n}}{2 \beta^{2} \sigma}\right) u_{t y y}\right\}_{k, j}^{n}(14) \\
\kappa_{5 x}=\alpha^{2} \Psi_{2 x} \sigma, \kappa_{5 y}=\beta^{2} \Psi_{2 y} \sigma, & +\left\{\kappa_{6} u_{x y}+\kappa_{7 x} u_{x x y}+\kappa_{7 y} u_{x y y}+\kappa_{8} u_{x x y y}\right\}_{k, j}^{n} \\
\kappa_{6}=-\frac{\alpha^{2}}{\beta}\left(\Psi_{1 x}-\alpha \Psi_{2 x} c_{1}\right) c_{2}-\frac{2 \alpha^{2} \Psi_{2 x}}{\beta}\left(c_{2}\right)_{x} & =\left\{-\frac{1}{\sigma}+\kappa_{9 x} \delta_{x}+\kappa_{10 x} \delta_{x}^{2}+\kappa_{9 y} \delta_{y}+\kappa_{10 y} \delta_{y}^{2}\right\} f_{k, j}^{n} \\
& +\frac{3 \kappa_{3}}{4 \sigma} f_{k, j}^{n}-\frac{\kappa_{3}}{\sigma} f_{k, j}^{n-1}+\frac{\kappa_{3}}{4 \sigma} f_{k, j}^{n-2}+O(\Delta) .
\end{array}
$$$$
-\frac{\beta^{2}}{\alpha}\left(\Psi_{1 y}-\beta \Psi_{2 y} c_{2}\right) c_{1}-\frac{2 \beta^{2} \Psi_{2 y}}{\alpha}\left(c_{1}\right)_{y}
$$$$
\kappa_{7 x}=-\frac{\alpha^{2} \Psi_{2 x}}{\beta} c_{2}-\frac{\beta^{2}}{\alpha^{2}}\left(\Psi_{1 y}-\beta \Psi_{2 y} c_{2}\right),
$$$$
\kappa_{7 y}=-\frac{\beta^{2} \Psi_{2 y}}{\alpha} c_{1}-\frac{\alpha^{2}}{\beta^{2}}\left(\Psi_{1 x}-\alpha \Psi_{2 x} c_{1}\right),
$$$$
\kappa_{8}=-\frac{\alpha^{2} \Psi_{2 x}}{\beta^{2}}-\frac{\beta^{2} \Psi_{2 y}}{\alpha^{2}},
$$$$
\kappa_{9 x}=\alpha^{2}\left(\Psi_{1 x}-\alpha \Psi_{2 x} c_{1}\right), \kappa_{9 y}=\beta^{2}\left(\Psi_{1 y}-\beta \Psi_{2 y} c_{2}\right),
$$$$
\kappa_{10 x}=\alpha^{2} \Psi_{2 x}, \kappa_{10 y}=\beta^{2} \Psi_{2 y} .
$$

The second-order derivative of time from Eq. (8) is $u_{t t}$ $=\left(1 / \alpha^{2} \sigma\right) u_{t x x}+\left(1 / \beta^{2} \sigma\right) u_{t y y}+\left(c_{1} / \alpha \sigma\right) u_{t x}+\left(c_{2} / \beta \sigma\right) u_{t y}+\left(f_{t} /\right.$ $\sigma)$. By using the forward difference scheme, we can get

$$
\begin{aligned}
\left(u_{t}\right)_{k, j}^{n} & =\frac{u_{k, j}^{n+1}-u_{k, j}^{n}}{\tau_{n}}-\frac{\tau_{n}}{2}\left(u_{t t}\right)_{k, j}^{n}+O\left(\tau^{2}\right) \\
& =\delta_{t}^{+} u_{k, j}^{n}-\frac{\tau_{n}}{2}\left(u_{t t}\right)_{k, j}^{n}+O\left(\tau^{2}\right) .
\end{aligned}
$$

Relying on the second-order backward Euler difference scheme, we can get

$$
\left(f_{t}\right)_{k, j}^{n}=\frac{3 f_{k, j}^{n}-4 f_{k, j}^{n-1}+f_{k, j}^{n-2}}{2 \tau_{n}}
$$

The first-order and the second-order derivate of $u$ with regard to space variables $x$ and $y$ are discretized by the central difference scheme. The first-order derivate of $u$ with regard to time variable $t$ is discretized by the forward difference scheme. Subsequently, after the difference approximations are carried out to $\left(u_{t x}\right)_{k, j}^{n},\left(u_{t x x}\right)_{k, j}^{n},\left(u_{t y}\right)_{k, j}^{n},\left(u_{t y y}\right)_{k, j}^{n},\left(u_{x y}\right)_{k, j}^{n}$, $\left(u_{x x y}\right)_{k, j}^{n},\left(u_{x y y}\right)_{k, j}^{n}$, and $\left(u_{x x y y}\right)_{k, j}^{n}$, a linear system is formed as

$$
\begin{aligned}
& b_{1 y} u_{k, j-1}^{n+1}+b_{1 x} u_{k-1, j}^{n+1}+b_{0} u_{k, j}^{n+1}+b_{2 x} u_{k+1, j}^{n+1}+b_{2 y} u_{k, j+1}^{n+1} \\
&= d_{1 y} u_{k, j-1}^{n}+d_{1 x} u_{k-1, j}^{n}+d_{0} u_{k, j}^{n}+d_{2 x} u_{k+1, j}^{n} \\
&+d_{2 y} u_{k, j+1}^{n}+d_{31} u_{k-1, j-1}^{n}+d_{32} u_{k+1, j-1}^{n}+d_{33} u_{k-1, j+1}^{n} \\
&+d_{34} u_{k+1, j+1}^{n}+s_{1 y} f_{k, j-1}^{n}+s_{1 x} f_{k-1, j}^{n}+s_{0} f_{k, j}^{n} \\
&+s_{2 x} f_{k+1, j}^{n}+s_{2 y} f_{k, j+1}^{n}+M_{1} f_{k, j}^{n-1}+M_{2} f_{k, j}^{n-2}
\end{aligned}
$$

where

$$
b_{1 y}=\frac{\left(-2 \tau_{n}+\beta c_{2} \tau_{n} y_{L}\right) \kappa_{3}-2 \beta^{2} \sigma y_{L} \kappa_{4 y}+4 \beta^{2} \sigma \kappa_{5 y}}{2 \beta^{2} \sigma \tau_{n} y_{L}\left(y_{R}+y_{L}\right)},
$$

$$
b_{1 x}=\frac{\left(2 \tau_{n}+\alpha c_{1} \tau_{n} x_{L}\right) \kappa_{3}-2 \alpha^{2} \sigma x_{L} \kappa_{4 x}+4 \alpha^{2} \sigma \kappa_{5 x}}{2 \alpha^{2} \sigma \tau_{n} x_{L}\left(x_{R}+x_{L}\right)}
$$$$
b_{0}=\frac{\kappa_{3}}{\tau_{n}}-\frac{2 \alpha^{2} \sigma \kappa_{5 x}-\tau_{n} \kappa_{3}}{\alpha^{2} \sigma \tau_{n} x_{R} x_{L}}-\frac{2 \beta^{2} \sigma \kappa_{5 y}-\tau_{n} \kappa_{3}}{\beta^{2} \sigma \tau_{n} y_{R} y_{L}},
$$$$
b_{2 x}=\frac{\left(-2 \tau_{n}-\alpha c_{1} \tau_{n} x_{R}\right) \kappa_{3}+2 \alpha^{2} \sigma x_{R} \kappa_{4 x}+4 \alpha^{2} \sigma \kappa_{5 x}}{2 \alpha^{2} \sigma \tau_{n} x_{R}\left(x_{R}+x_{L}\right)},
$$$$
b_{2 y}=\frac{\left(-2 \tau_{n}-\beta c_{2} \tau_{n} y_{R}\right) \kappa_{3}+2 \beta^{2} \sigma y_{R} \kappa_{4 y}+4 \beta^{2} \sigma \kappa_{5 y}}{2 \beta^{2} \sigma \tau_{n} y_{R}\left(y_{R}+y_{L}\right)},
$$ 


$$
\begin{array}{rlrl}
d_{1 y}= & \frac{\kappa_{1 y}}{y_{R}+y_{L}}-\frac{2 \kappa_{2 y}}{y_{L}\left(y_{R}+y_{L}\right)} & s_{1 y}=-\frac{\kappa_{9 y}}{y_{R}+y_{L}}+\frac{2 \kappa_{10 y}}{y_{L}\left(y_{R}+y_{L}\right)}, \\
& +\frac{\left(-2 \tau_{n}+\beta c_{2} \tau_{n} y_{L}\right) \kappa_{3}-2 \beta^{2} \sigma y_{L} \kappa_{4 y}+4 \beta^{2} \sigma \kappa_{5 y}}{2 \beta^{2} \sigma \tau_{n} y_{L}\left(y_{R}+y_{L}\right)} & s_{1 x}=-\frac{\kappa_{9 x}}{x_{R}+x_{L}}+\frac{2 \kappa_{10 x}}{x_{L}\left(x_{R}+x_{L}\right)}, \\
& -\frac{2 \kappa_{7 x}}{x_{R} x_{L}\left(y_{R}+y_{L}\right)}+\frac{4 \kappa_{8}}{x_{R} x_{L} y_{L}\left(y_{R}+y_{L}\right)}, & s_{0}=\frac{3 \kappa_{3}-4}{4 \sigma}-\frac{2 \kappa_{10 x}}{x_{R} x_{L}}-\frac{2 \kappa_{10 y}}{y_{R} y_{L}}, \\
d_{1 x}= & \frac{\kappa_{1 x}}{x_{R}+x_{L}}-\frac{2 \kappa_{2 x}}{x_{L}\left(x_{R}+x_{L}\right)} & s_{2 x}=\frac{\kappa_{9 x}}{x_{R}+x_{L}}+\frac{2 \kappa_{10 x}}{x_{R}\left(x_{R}+x_{L}\right)}, \\
& +\frac{\left(-2 \tau_{n}+\alpha c_{1} \tau_{n} x_{L}\right) \kappa_{3}-2 \alpha^{2} \sigma x_{L} \kappa_{4 x}+4 \alpha^{2} \sigma \kappa_{5 x}}{2 \alpha^{2} \sigma \tau_{n} x_{L}\left(x_{R}+x_{L}\right)} & \frac{4 \kappa_{8}}{2 \kappa_{7 y}}+\frac{\left.x_{R}+x_{L}\right) y_{R} y_{L}}{x_{L}\left(x_{R}+x_{L}\right) y_{R} y_{L}}, & s_{2 y}=\frac{\kappa_{9 y}}{y_{R}+y_{L}}+\frac{2 \kappa_{10 y}}{y_{R}\left(y_{R}+y_{L}\right)}, \\
d_{0}= & \frac{2 \kappa_{2 x}}{x_{R} x_{L}}+\frac{2 \kappa_{2 y}}{y_{R} y_{L}}+\frac{\kappa_{3}}{\tau_{n}}-\frac{2 \alpha^{2} \sigma \kappa_{5 x}-\tau_{n} \kappa_{3}}{\alpha^{2} \sigma \tau_{n} x_{R} x_{L}} & M_{1}=-\frac{\kappa_{3}}{\sigma}, M_{2}=\frac{\kappa_{3}}{4 \sigma} .
\end{array}
$$$$
-\frac{2 \beta^{2} \sigma \kappa_{5 y}-\tau_{n} \kappa_{3}}{\beta^{2} \sigma \tau_{n} y_{R} y_{L}}-\frac{4 \kappa_{8}}{x_{R} x_{L} y_{R} y_{L}}
$$$$
d_{2 x}=\frac{\kappa_{1 x}}{x_{R}+x_{L}}-\frac{2 \kappa_{2 x}}{x_{R}\left(x_{R}+x_{L}\right)}
$$$$
\frac{\left(-2 \tau_{n}-\alpha c_{1} \tau_{n} x_{R}\right) \kappa_{3}+2 \alpha^{2} \sigma x_{R} \kappa_{4 x}+4 \alpha^{2} \sigma \kappa_{5 x}}{2 \alpha^{2} \sigma \tau_{n} x_{R}\left(x_{R}+x_{L}\right)}
$$$$
+\frac{2 \kappa_{7 y}}{\left(x_{R}+x_{L}\right) y_{R} y_{L}}+\frac{4 \kappa_{8}}{x_{R}\left(x_{R}+x_{L}\right) y_{R} y_{L}} \text {, }
$$$$
d_{2 y}=-\frac{\kappa_{1 y}}{y_{R}+y_{L}}-\frac{2 \kappa_{2 y}}{y_{R}\left(y_{R}+y_{L}\right)}
$$$$
+\frac{\left(-2 \tau_{n}-\beta c_{2} \tau_{n} y_{R}\right) \kappa_{3}+2 \beta^{2} \sigma y_{R} \kappa_{4 y}+4 \beta^{2} \sigma \kappa_{5 y}}{2 \beta^{2} \sigma \tau_{n} y_{R}\left(y_{R}+y_{L}\right)}
$$$$
+\frac{2 \kappa_{7 x}}{x_{R} x_{L}\left(y_{R}+y_{L}\right)}+\frac{4 \kappa_{8}}{x_{R} x_{L} y_{R}\left(y_{R}+y_{L}\right)} \text {, }
$$$$
d_{31}=-\frac{\kappa_{6}}{\left(x_{R}+x_{L}\right)\left(y_{R}+y_{L}\right)}+\frac{2 \kappa_{7 x}}{x_{L}\left(x_{R}+x_{L}\right)\left(y_{R}+y_{L}\right)}
$$$$
+\frac{2 \kappa_{7 y}}{\left(x_{R}+x_{L}\right) y_{L}\left(y_{R}+y_{L}\right)}-\frac{4 \kappa_{8}}{x_{L}\left(x_{R}+x_{L}\right) y_{L}\left(y_{R}+y_{L}\right)} \text {, }
$$$$
d_{32}=\frac{\kappa_{6}}{\left(x_{R}+x_{L}\right)\left(y_{R}+y_{L}\right)}+\frac{2 \kappa_{7 x}}{x_{R}\left(x_{R}+x_{L}\right)\left(y_{R}+y_{L}\right)}
$$$$
-\frac{2 \kappa_{7 y}}{\left(x_{R}+x_{L}\right) y_{L}\left(y_{R}+y_{L}\right)}-\frac{4 \kappa_{8}}{x_{R}\left(x_{R}+x_{L}\right) y_{L}\left(y_{R}+y_{L}\right)} \text {, }
$$$$
d_{33}=\frac{\kappa_{6}}{\left(x_{R}+x_{L}\right)\left(y_{R}+y_{L}\right)}-\frac{2 \kappa_{7 x}}{x_{L}\left(x_{R}+x_{L}\right)\left(y_{R}+y_{L}\right)}
$$$$
+\frac{2 \kappa_{7 y}}{\left(x_{R}+x_{L}\right) y_{R}\left(y_{R}+y_{L}\right)}-\frac{4 \kappa_{8}}{x_{L}\left(x_{R}+x_{L}\right) y_{R}\left(y_{R}+y_{L}\right)} \text {, }
$$$$
d_{34}=-\frac{\kappa_{6}}{\left(x_{R}+x_{L}\right)\left(y_{R}+y_{L}\right)}-\frac{2 \kappa_{7 x}}{x_{R}\left(x_{R}+x_{L}\right)\left(y_{R}+y_{L}\right)}
$$$$
-\frac{2 \kappa_{7 y}}{\left(x_{R}+x_{L}\right) y_{R}\left(y_{R}+y_{L}\right)}-\frac{4 \kappa_{8}}{x_{R}\left(x_{R}+x_{L}\right) y_{R}\left(y_{R}+y_{L}\right)} \text {, }
$$

Through the prior deducing procedure, it can be seen that the truncation error of the Eq. (15) is-

$O\left(\tau_{n}^{2}+\tau_{n}\left(x_{R}^{2}-x_{R} x_{L}+x_{L}^{2}\right)+\tau_{n}\left(y_{R}^{2}-y_{R} y_{L}+y_{L}^{2}\right)+\left(x_{R}^{5}+x_{L}^{5} / x_{R}\right.\right.$ $\left.\left.+x_{L}\right)+\left(y_{R}^{5}+y_{L}^{5} / y_{R}+y_{L}\right)\right)$. When $h=x_{R}=x_{L}=y_{R}=y_{L}$ and $\tau_{n}=h^{2}$, it owns spatial accuracy of fourth order and temporal accuracy of second order.

\section{Adaptive Grid Structure}

3.1. Adaptive Grid Structure in Time. The adaptation mesh technique in the temporal and the spatial direction is employed for solving the $2 \mathrm{D}$ problem of quenching type. Obviously, the 2D adaptive mesh algorithm can be derived from the $1 \mathrm{D}$ case. The arc-length monitor function of the temporal derivative function resulting from equal distribution principle is used to design the temporal and the spatial moving mesh algorithm, respectively. From Ref. [19, 20], a self-adaptive grid in the time direction is given as follows. A maximized ratio equation for computing the adaptive temporal interval $\tau_{n}$ is

$$
\frac{\tau_{n}}{\tau_{n-1}}=\frac{\max _{\substack{0<k<K \\ 0<j<J}} \sqrt{1+\left[\left(\partial^{2} u / \partial t^{2}\right)\left(x_{k}, y_{j}, t_{n-1 / 2}\right)\right]^{2}}}{\max _{\substack{0<k<K \\ 0<j<J}} \sqrt{1+\left[\left(\partial^{2} u / \partial t^{2}\right)\left(x_{k}, y_{j}, t_{n+1 / 2}\right)\right]^{2}}}, n=1,2, \cdots
$$

The standard uniform central difference formula is substituted for the $\partial^{2} u / \partial t^{2}$ in Eq. (17). Of course, when the prior temporal step $\tau_{n-1}$ is given, the current temporal step $\tau_{n}$ can be calculated through Eq. (17).

3.2. Adaptive Grid Structure in Space. Let a spatial adaptation algorithm be deduced as follows. $W_{x}(x, t)$ is taken as the monitor function in $x$-direction and $W_{y}(y, t)$ is taken as the monitor function in $y$ - direction. 
$W_{x}(x, t)=\sqrt{1+\sum_{k=0}^{K}\left[\left(\partial^{2} u / \partial t \partial x\right)(x, t)\right]^{2}}, 0 \leq x \leq 1,0 \leq t<T$,

$W_{y}(y, t)=\sqrt{1+\sum_{j=0}^{J}\left[\left(\partial^{2} u / \partial t \partial y\right)(y, t)\right]^{2}}, 0 \leq y \leq 1,0 \leq t<T$.

$\left\{\xi_{k}\left(x_{k}, t\right)\right\}_{k=0}^{K}$ and $\left\{\zeta_{j}\left(y_{j}, t\right)\right\}_{j=0}^{J}$ point to the set of $2 \mathrm{D}$ original spatial mesh points in $x$-direction and $y$-direction. The $2 \mathrm{D}$ computational area is transformed as $\left\{x_{k}\left(\xi_{k}, t\right)\right\}_{k=0}^{K}$ in $x$-direction and $\left\{y_{j}\left(\zeta_{j}, t\right)\right\}_{j=0}^{J}$ in $y$-direction through mesh movement. The continuous function $W_{x}(x, t)>0$ and $W_{y}(y, t)>0$ in the definition area $(x, y) \epsilon$ $(0,1) \times(0,1)$ equally distributes over the $2 \mathrm{D}$ mesh refreshed. In fact, the $1 \mathrm{D}$ adaptive mesh algorithm individually carries out in each spatial direction. According to [19, 20], we can get the semi-implicit scheme in $x$-direction

$$
\begin{aligned}
& W_{x}\left(x_{k+1 / 2}^{(m)}, t_{n}\right)\left(x_{k+1}^{(m+1)}-x_{k}^{(m+1)}\right) \\
& \quad-W_{x}\left(x_{k-1 / 2}^{(m)}, t_{n}\right)\left(x_{k}^{(m+1)}-x_{k-1}^{(m+1)}\right) \\
& =0, k=1,2, \cdots, K-1, n \geq 0 .
\end{aligned}
$$

Similarly, we can get the semi-implicit scheme in $y-$ direction

$$
\begin{aligned}
& W_{y}\left(y_{j+1 / 2}^{(m)}, t_{n}\right)\left(y_{j+1}^{(m+1)}-y_{j}^{(m+1)}\right) \\
& \quad-W_{y}\left(y_{j-1 / 2}^{(m)}, t_{n}\right)\left(y_{j}^{(m+1)}-y_{j-1}^{(m+1)}\right) \\
& =0, j=1,2, \cdots, J-1, n \geq 0 .
\end{aligned}
$$

3.3. Iterative Adaption Algorithm. This paragraph shows the iterative process of the presented method in the paper.

Step 1. Depending on the spatial mesh $\left\{x_{k}^{(m)}\right\}_{k=0}^{K},\left\{y_{j}^{(m)}\right\}_{j=0}^{J}$ at the $n$th time layer, we can obtain the corresponding monitor functions $W_{x}(x, t)$ and $W_{y}(y, t)$ via Eqs. (18) and (19).

Step 2. The point set $\left\{x_{k}^{(m+1)}\right\}_{k=0}^{K}$ refreshed is extracted from the prior grid $\left\{x_{k}^{(m)}\right\}_{k=0}^{K}$ via Eq. (20) whereas $\left\{y_{j}^{(m+1)}\right\}_{j=0}^{J}$ refreshed is extracted from $\left\{y_{j}^{(m)}\right\}_{j=0}^{J}$ by Eq. (21). We use the new point sets to get the replacement for the old point sets iteratively once $\sum_{k=1}^{K-1}\left\|x_{k}^{(m+1)}-x_{k}^{(m)}\right\|<e r, \sum_{j=1}^{J-1}\left\|y_{j}^{(m+1)}-y_{j}^{(m)}\right\|<$ $e r$, which er takes $10^{-5}$. The last value of $m+1$ takes $M_{n}$.

Step 3. By virtue of the last grid point sets $\left\{x_{k}^{\left(M_{n}\right)}\right\}_{k=0}^{K}$, $\left\{y_{j}^{\left(M_{n}\right)}\right\}_{j=0}^{J}$, and area ratio algorithm, the solutions in the $n$ th time line $\left\{u_{k, j}^{n}\right\}_{k=1, j=1}^{K-1, J-1}$ are all estimated. Combining with $\tau_{n}$ obtained from Eq. (17), $\left\{u_{k, j}^{n+1}\right\}_{k=1, j=1}^{K-1, J-1}$ in the $n+1$ th time line can be calculated by Eq. (15).

Step 4. Repeat steps from the first to the third till quenching occurs, or the solution converges to a steady solution.

\section{Simulation Demonstration}

4.1. Numerical Case. This is a common numerical sample utilized to evaluate the performance of Eq. (15) measure by Eq. (22)

$$
\begin{aligned}
p u_{t}=u_{x x}+ & u_{y y}-p u_{x}-p u_{y},(x, y) \in[0,1] \times[0,1], t \geq 0 \\
u(x, y, t)= & \frac{e^{p x}-1}{e^{p}-1}+\frac{e^{p y}-1}{e^{p}-1} \\
& +\sum_{q=1}^{\infty} \frac{(-1)^{q} q \pi}{(q \pi)^{2}+p^{2} / 4} e^{p(x-1) / 2+p(y-1) / 2} \sin \\
& \cdot(q \pi x+q \pi y) e^{-\left[(q \pi)^{2} / p+p / 4\right] t}
\end{aligned}
$$

In the paper, $T=0.5, \tau_{n}=0.01, p=100$. The initial and boundary values can be computed through the exact solution. The tested results display in Table 1, in which there are four criteria containing Max. error, Aver. error, CPU time, and Conv. rate. Max. error means the maximal absolute error between analysis solution and difference solution; Aver. error means the average absolute error; CPU time means the running time of system; Conv. rate means convergence rate between the two interfacing mesh numbers. The nonuniform mesh follows the rule: $\left\{k / K+\left(\lambda_{x} / \pi\right) \sin (\pi k / K)\right\}_{k=0}^{K}$ in $x-$ direction and $\left\{j / J+\left(\lambda_{y} / \pi\right) \sin (\pi j / J)\right\}_{j=0}^{J}$ in $y$-direction, where $K$ and $J$ point to intervals of spatial direction and $K$ $=J$, whereas $\lambda_{x}$ and $\lambda_{y}$ mean telescopic transformation coefficient and $\lambda_{x}=\lambda_{y}$. There exist six difference schemes that are compared on another for the example in Table 1, which are the schemes in Ref. [26] on the uniform and the nonuniform grid, the schemes in Ref. [27] on the uniform and the nonuniform grid, and the proposed schemes on the uniform and the nonuniform grid. The schemes in Ref. [27] and the proposed schemes all chose the five spatial intervals $16,32,64,128,256$, and $\lambda_{x}=\lambda_{y}=0.7$ and the four aforementioned criteria. Only the schemes in Ref. [26] choose the five spatial intervals 16, $32,64,128$, and $\lambda_{x}=\lambda_{y}=0.4$ and the three aforementioned criteria with the exception of Aver. error.

With regard to Max. error and Aver. error, the schemes on the uniform grid are inferior to the schemes on the nonuniform grid; the proposed schemes are superior to the other schemes, and the schemes (nonuniform) in Ref. [27] are superior to the scheme (nonuniform) in Ref. [26], and the tendency becomes more obviously as $K(J)$ rises. When $K(J$ ) is 128 , the maximal absolute error of the scheme in Ref. [27] (uniform) is $3.1469 \times 10^{-4}$, and the maximal absolute error of the scheme in Ref. [27] (nonuniform) is $9.3004 \times$ $10^{-7}$; the maximal absolute error of the scheme in Ref. [26] 
TABLE 1: The parameter values for common example.

\begin{tabular}{|c|c|c|c|c|c|}
\hline Schemes & $K(J)$ & Max. error & Aver. error & CPU time & Conv. rate \\
\hline \multirow{4}{*}{ The scheme in Ref. [26] (uniform) } & 16 & $2.59 \times 10^{-1}$ & - & 1000 & - \\
\hline & 32 & $5.65 \times 10^{-2}$ & - & 1344 & 2.30 \\
\hline & 64 & $5.50 \times 10^{-3}$ & - & 6766 & 3.44 \\
\hline & 128 & $3.15 \times 10^{-4}$ & & 61328 & 4.17 \\
\hline \multirow{4}{*}{ The scheme in Ref. [26] (nonuniform) } & 16 & $1.12 \times 10^{-1}$ & - & 844 & - \\
\hline & 32 & $1.22 \times 10^{-2}$ & - & 1625 & 3.34 \\
\hline & 64 & $6.84 \times 10^{-4}$ & - & 11188 & 4.25 \\
\hline & 128 & $4.42 \times 10^{-5}$ & - & 135344 & 4.00 \\
\hline \multirow{5}{*}{ The scheme in Ref. [27] (uniform) } & 16 & $2.4859 \times 10^{-1}$ & $1.7858 \times 10^{-2}$ & 47 & - \\
\hline & 32 & $5.7032 \times 10^{-2}$ & $1.9148 \times 10^{-3}$ & 172 & 5.0033 \\
\hline & 64 & $5.5382 \times 10^{-3}$ & $1.4565 \times 10^{-4}$ & 702 & 3.7166 \\
\hline & 128 & $3.1469 \times 10^{-4}$ & $9.6850 \times 10^{-6}$ & 2948 & 3.9106 \\
\hline & 256 & $1.9710 \times 10^{-5}$ & $6.1742 \times 10^{-7}$ & 11794 & 3.9714 \\
\hline \multirow{5}{*}{ The scheme in Ref. [27] (nonuniform) } & 16 & $8.1898 \times 10^{-3}$ & $8.7725 \times 10^{-4}$ & 358 & - \\
\hline & 32 & $2.9724 \times 10^{-4}$ & $2.9996 \times 10^{-5}$ & 2855 & 7.9861 \\
\hline & 64 & $1.5573 \times 10^{-5}$ & $1.6521 \times 10^{-6}$ & 14914 & 4.1824 \\
\hline & 128 & $9.3004 \times 10^{-7}$ & $1.0224 \times 10^{-7}$ & 35006 & 4.1964 \\
\hline & 256 & $1.3364 \times 10^{-7}$ & $6.4492 \times 10^{-9}$ & 165969 & 4.7029 \\
\hline \multirow{5}{*}{ The proposed scheme (uniform) } & 16 & $2.5865 \times 10^{-1}$ & $1.7571 \times 10^{-2}$ & 78 & - \\
\hline & 32 & $5.6511 \times 10^{-2}$ & $1.9107 \times 10^{-3}$ & 297 & 4.9902 \\
\hline & 64 & $5.5041 \times 10^{-3}$ & $1.4562 \times 10^{-4}$ & 1217 & 3.7138 \\
\hline & 128 & $3.1447 \times 10^{-4}$ & $9.6804 \times 10^{-6}$ & 5148 & 3.9110 \\
\hline & 256 & $1.9357 \times 10^{-5}$ & $5.9061 \times 10^{-7}$ & 20951 & 4.0348 \\
\hline \multirow{5}{*}{ The proposed scheme (nonuniform) } & 16 & $8.1405 \times 10^{-3}$ & $8.7581 \times 10^{-4}$ & 421 & - \\
\hline & 32 & $2.9716 \times 10^{-4}$ & $2.9989 \times 10^{-5}$ & 1857 & 7.9849 \\
\hline & 64 & $1.5491 \times 10^{-5}$ & $1.6490 \times 10^{-6}$ & 6911 & 4.1847 \\
\hline & 128 & $6.9033 \times 10^{-7}$ & $8.9949 \times 10^{-8}$ & 24585 & 4.1964 \\
\hline & 256 & $4.1212 \times 10^{-8}$ & $3.4536 \times 10^{-9}$ & 131243 & 4.7029 \\
\hline
\end{tabular}

(uniform) is $3.15 \times 10^{-4}$, and the maximal absolute error of the scheme in Ref. [26] (nonuniform) is $4.42 \times 10^{-5}$; the maximal absolute error of the proposed scheme (uniform) is $3.1447 \times 10^{-4}$, and the maximal absolute error of the proposed scheme (nonuniform) is $6.9033 \times 10^{-7}$. When $K(J)$ is 256, the average error of the scheme in Ref. [27] (uniform) is $6.1742 \times 10^{-7}$, and the average error of the scheme in Ref. [27] (nonuniform) is $6.4492 \times 10^{-9}$; the average error of the proposed scheme (uniform) is $5.9061 \times 10^{-7}$, and the average error of the proposed scheme (nonuniform) is $3.4536 \times 10^{-9}$. In terms of CPU time, the scheme (nonuniform) in Ref. [27] spends the most time among the schemes when $K(J)$ is 32 , 64,128 , and 256, and the tendency becomes more obviously as $K(J)$ rises; the schemes in Ref. [26] spend much more time than the proposed schemes do. Typically, the running time of the schemes on the uniform grid is less than that of the schemes on the nonuniform grid. It just means that the latter complies with a time-for-space rule, which refers to it may improve the spatial accuracy of an algorithm by adding its running time.

4.2. Quenching Case without Convection Term. When $c_{1}(x$, $y)=0$ and $c_{2}(x, y)=0$, with the initial and boundary conditions Eqs. (6) and (7), the original Eq. (5) can be described as

$$
\begin{aligned}
\left(\alpha^{2} x^{2}+\beta^{2} y^{2}\right)^{q / 2} u_{t}= & \frac{1}{\alpha^{2}} u_{x x}+\frac{1}{\beta^{2}} u_{y y} \\
& +\frac{1}{(1-u)^{\theta}},(x, y) \in(0,1) \\
& \times(0,1), t \in(0, T)
\end{aligned}
$$


TABLE 2: Quenching performances of different schemes for $q=0, a=\beta=\sqrt{10}$, and $\theta=1$ without convection term.

\begin{tabular}{lccccc}
\hline Schemes & $x_{\max }$ & $y_{\max }$ & $T_{\max }$ & $\max u$ & $\max u_{t}$ \\
\hline The proposed scheme & 0.500 & 0.500 & 0.5990174307488624 & 0.9901182463562488 & 83.20947696438735 \\
The scheme in [19] & - & - & 0.58712499993751 & 0.990432 & 148.887767 \\
The scheme in [20] & 0.500 & 0.500 & 0.587554 & 0.999263 & 1249.917563 \\
\hline
\end{tabular}

For the situation of $c_{1}(x, y)=0$ and $c_{2}(x, y)=0$, we take advantage of Eq. (15) to approximate Eq. (24) and gain quenching case without convection term. We set the three parameters $q=0, a=\beta=\sqrt{10}$, and $\theta=1$ to the case which had been illustrated in Refs $[19,20]$. In this case, the initial temporal step is configured as $\tau_{0}=4.166677 \times 10^{-5}$, and the initial space step is configured as $h_{0}=1 / 90$.

Table 2 offers quenching performances of the three different schemes, which are from Refs $[19,20]$ besides the proposed method on adaptive mesh, for $q=0, a=\beta=\sqrt{10}$, and $\theta=1$ without convection term. The criteria in Table 2 are explained as follows. $\left(x_{\max }, y_{\max }\right)$ refers to the point of quenching location, $T_{\max }$ refers to the quenching time, $\max u$ refers to the maximum temperature value immediately before quenching happens, and $\max u_{t}$ refers to the maximum variation of temperature with respect to time immediately before quenching happens. For the three schemes, their quenching locations are all $(0.5,0.5)$. There are subtle differences among the three schemes for quenching time and $\max u . T_{\max }$ of the proposed scheme is 0.5990174307488624 , and max $u$ of the proposed scheme is 0.9901182463562488 . But there are greater differences among the three schemes for $\max u_{t}$. $\max u_{t}$ of the proposed scheme is 83.20947696438735 while those of the schemes in Refs $[19,20]$ are 148.887767 and 1249.917563. There is no effect of the differences on quenching research. Figure 1 offers the three-dimensional scenes of $u$ and $u_{t}$ with regard to spatial variables $x, y$ when the time $t$ is 0.5990174307488624 , respectively. From the two 3D surfaces at the time $T_{\max }=0.5990174307488624$ that is immediately before quenching occurs, it is found that $u \longrightarrow 1^{-}$and $u_{t}$ $\longrightarrow 84^{-}$at the quenching location $\left(x_{\max }, y_{\max }\right)=(0.5,0.5)$ and the quenching time $T_{\max }=0.5990174307488624$.

4.3. Quenching Case with Convection Term $b /(1+\alpha x+\beta y)$. We will investigate what role degeneration parameter $q$, convection parameter $b$, and combustion chamber size $\alpha(\beta)$ in Eqs. (5)-(7) play during the quenching process and enumerate the representative Case 5.0 to show special quenching features when $c_{1}(x, y)=b_{x} /(1+\alpha x+\beta y) \quad$ and $c_{2}(x, y)=b_{y} /(1+\alpha x+\beta y)$, where $b_{x}$ and $b_{y}$ take the constants and convection parameter $b=b_{x}=b_{y}$. When singularity parameter $\theta$ is larger than 1 , the quenching situation is complex. Therefore, we only consider quenching cases of $\theta$ $=1$. In numerical samples, $b_{x}$ and $b_{y}$ range from -200 to 200; $\alpha^{2}$ range from 15 to 300000 . These are the same to Section 4.4. To better illustrate the problems, we choose some representative data from lots of tests to discuss quenching phenomena in the next paragraphs. For the convection model $b /(1+\alpha x+\beta y)$, we set $h_{0}=1 / 70$ as the initial $x$ $-(y-)$ step and $\tau_{0}=0.0008$ as the initial $t$-step.
Tables 3-6 offer some typical quenching cases to demonstrate the relationship between the three groups of parameters and quenching phenomena. Case 5.0 recorded as a reference in Table 3, i.e., $q=1, b_{x}=b_{y}=3, \alpha=\beta=15$, is compared with other cases in Tables $3-5$. Table 6 displays the specific quenching data of Case 5.0. Figures 1-3 show the quenching statuses of Case 5.0 relative to $u, u_{t}, x, y$, and $t$.

Except Case 5.0, there are ten quenching cases chosen in Table 3. The ten items list as follows: Case 5.1.1 is $q=0.1$, $b_{x}=b_{y}=3, \alpha=\beta=15$; Case 5.1 .2 is $q=0.2, b_{x}=b_{y}=3, \alpha=\beta$ $=15$; Case 5.1.3 is $q=0.4, b_{x}=b_{y}=3, \alpha=\beta=15$; Case 5.1.4 is $q=0.6, b_{x}=b_{y}=3, \alpha=\beta=15$; Case 5.1 .5 is $q=0.7, b_{x}=$ $b_{y}=3, \alpha=\beta=15$; Case 5.1.6 is $q=0.9, b_{x}=b_{y}=3, \alpha=\beta=$ 15; Case1.1.7 is $q=1.1, b_{x}=b_{y}=3, \alpha=\beta=15$; Case1.1.8 is $q$ $=1.2, b_{x}=b_{y}=3, \alpha=\beta=15$; Case 5.1 .9 is $q=1.3, b_{x}=b_{y}=$ $3, \alpha=\beta=15$; Case 5.1 .10 is $q=1.4, b_{x}=b_{y}=3, \alpha=\beta=15$. Although the program may run when $q \geq 1.5$, it is hard to form quenching behaviors. If $q$ belongs to the definition domain $[0.1,1.4]$, the quenching phenomena will occur. From serial numerical cases, we can easily see the performances of quenching location and time. The quenching location $\left(x_{\max }, y_{\max }\right)$ declines as $q$ evolves and finally converges to $(0.04285714285714286,0.04285714285714286)$. There is an intermediate point $q=0.9$ dividing the definition domain as $[0.1,0.9]$ and $[1.0,1.4]$. The quenching location $x_{\max }$ and $y_{\max }$ do decline as $q$ evolves in the first subdomain and converge to $(0.04285714285714286,0.04285714285714286)$ in the second subdomain. The quenching time $T_{\max }$ reaches the maximum 9.686902288384093 when $q=1.4 . T_{\max }$ and qare in positive proportion when $q$ in $[0.1,1.4]$.

We choose ten items written in Table 4 to display the quenching characteristics related to the parameters $b_{x}$ and $b_{y}$. Case 5.2.1 is $q=1, b_{x}=b_{y}=-5, \alpha=\beta=15$; Case 5.2.2 is $q=1, b_{x}=b_{y}=-3, \alpha=\beta=15 ; \quad$ Case $\quad 5.2 .3$ is $q=1, b_{x}=b_{y}=0, \alpha=\beta=15$; Case 5.2 .4 is $q=1, b_{x}=b_{y}=2$ , $\alpha=\beta=15$; Case 5.2 .5 is $q=1, b_{x}=b_{y}=4, \alpha=\beta=15$; Case 5.2.6 is $q=1, b_{x}=b_{y}=5, \alpha=\beta=15$; Case 5.2 .7 is $q=1, b_{x}=$ $b_{y}=6, \alpha=\beta=15$; Case 5.2 .8 is $q=1, b_{x}=b_{y}=8, \alpha=\beta=15$; Case 5.2.9 is $q=1, b_{x}=b_{y}=9, \alpha=\beta=15$; Case 5.2.10 is $q=$ $1, b_{x}=b_{y}=10, \alpha=\beta=15 ; b_{x}$ and $b_{y}$ in Eq. (5) are theoretically equivalent, i.e., $b=b_{x}=b_{y}$. A valid scope of $b$ is $[-5,10$ ] for these cases. Because quenching occurs fastest when $b$ takes $4, b=4$ is thought as a special point for quenching time. Quenching time is inversely proportional to $b$ when $b \in[-5$ $, 4]$ and positively proportional to $b$ when $b \in[4,10]$. Quenching location $\left(x_{\max }, y_{\max }\right)$ rises as $b$ increases when $b \in[-5,5]$ and takes $(0.02857142857142857,0.02857142857142857)$ when $b \geq 6$. 


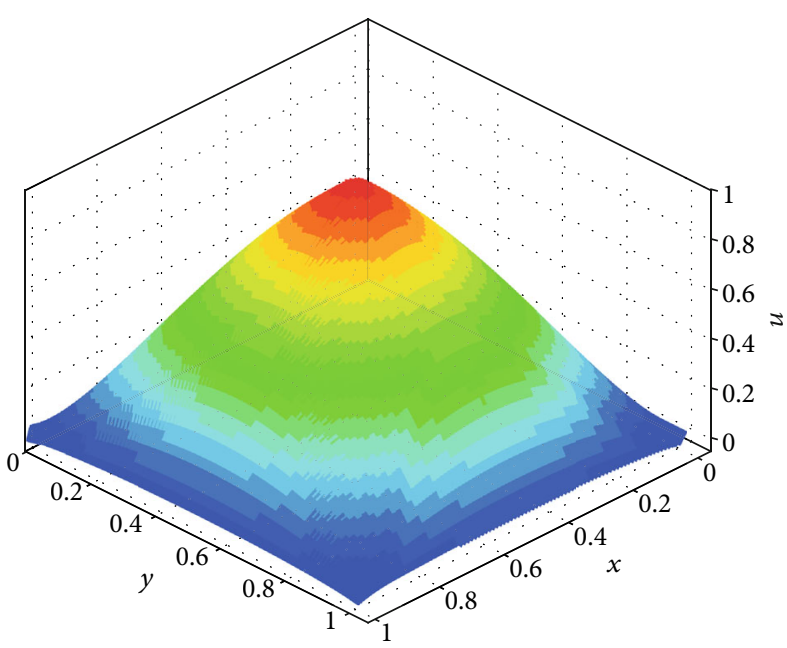

(a)

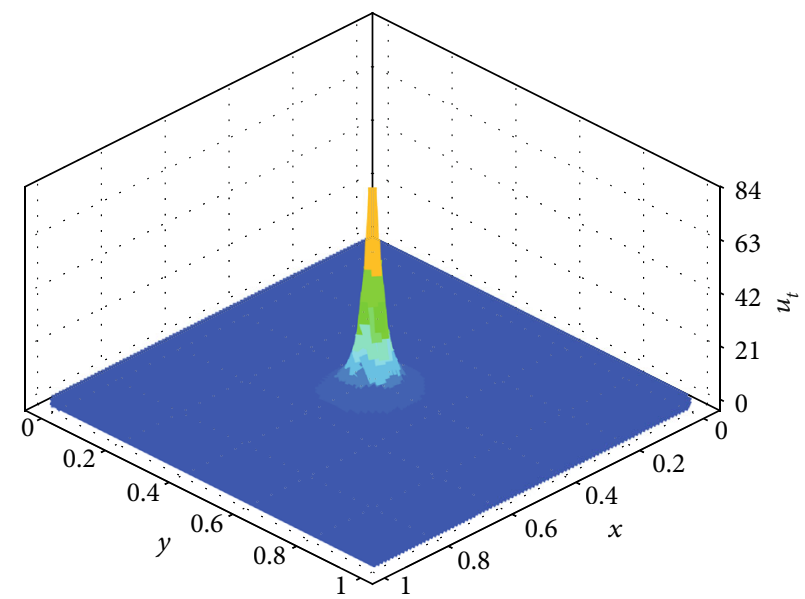

(b)

Figure 1: (a) The 3D plots of $u$ immediately before quenching occurs, and (b) the 3D plots of $u_{t}$ immediately before quenching occurs. The parameters are $q=0, a=\beta=\sqrt{10}$, and $\theta=1$ without convection term.

TABLE 3: Quenching data of $q$ based on convection term $b /(1+\alpha x+\beta y)$.

\begin{tabular}{lccccc}
\hline Case name & $x_{\max }$ & $y_{\max }$ & $T_{\max }$ & $\max u$ & $\max u_{t}$ \\
\hline Case 5.0 & 0.04285714285714286 & 0.04285714285714286 & 3.428404873627137 & 0.9920620807720361 & 125.6265973017968 \\
Case 5.1.1 & 0.1 & 0.1 & 0.6470842629145379 & 0.9933649818060033 & 136.4723853886283 \\
Case 5.1.2 & 0.08571428571428572 & 0.08571428571428572 & 0.7824847046607435 & 0.9903819718639215 & 87.67058865539519 \\
Case 5.1.3 & 0.07142857142857143 & 0.07142857142857143 & 1.109942679446164 & 0.9908479463229512 & 86.47536110256003 \\
Case 5.1.4 & 0.05714285714285714 & 0.05714285714285714 & 1.572860422734615 & 0.9902786764164395 & 83.87879039070892 \\
Case 5.1.5 & 0.05714285714285714 & 0.05714285714285714 & 1.88098095097722 & 0.9920140466370957 & 101.1277413288689 \\
Case 5.1.6 & 0.05714285714285714 & 0.05714285714285714 & 2.771928557465351 & 0.9920059842963465 & 96.8866420271182 \\
Case 5.1.7 & 0.04285714285714286 & 0.04285714285714286 & 4.293156056984269 & 0.9908925785572684 & 107.7822215514385 \\
Case 5.1.8 & 0.04285714285714286 & 0.04285714285714286 & 5.485318052886954 & 0.9920945867539992 & 126.7837317275917 \\
Case 5.1.9 & 0.04285714285714286 & 0.04285714285714286 & 7.182200512059787 & 0.9903718441627313 & 102.1421834048094 \\
Case 5.1.10 & 0.04285714285714286 & 0.04285714285714286 & 9.686902288384093 & 0.992048580687276 & 127.8241083054763 \\
\hline & & & & &
\end{tabular}

TABLE 4: Quenching data of $b_{x}$ and $b_{y}$ based on convection term $b /(1+\alpha x+\beta y)$.

\begin{tabular}{lccccc}
\hline Case name & $x_{\max }$ & $y_{\max }$ & $T_{\max }$ & $\max$ & $u$ \\
\hline Case 5.2.1 & 0.1714285714285714 & 0.1714285714285714 & 8.379050080786115 & 0.990231496274097 & 27.01166111192879 \\
Case 5.2.2 & 0.1285714285714286 & 0.1285714285714286 & 6.144661979620801 & 0.9902084486956095 & 35.54184621151833 \\
Case 5.2.3 & 0.08571428571428572 & 0.08571428571428572 & 4.133262574622704 & 0.9902265728041954 & 52.5010571873496 \\
Case 5.2.4 & 0.05714285714285714 & 0.05714285714285714 & 3.540661768514728 & 0.9901040464540505 & 75.11254034765163 \\
Case 5.2.5 & 0.04285714285714286 & 0.04285714285714286 & 3.389328541055185 & 0.992283726259128 & 128.3227508596781 \\
Case 5.2.6 & 0.04285714285714286 & 0.04285714285714286 & 3.449401822644199 & 0.9910523423231372 & 108.73967781816 \\
Case 5.2.7 & 0.02857142857142857 & 0.02857142857142857 & 3.578376058086737 & 0.990585829058986 & 147.5334079485446 \\
Case 5.2.8 & 0.02857142857142857 & 0.02857142857142857 & 4.018879993939997 & 0.9940259443236479 & 246.3627497716133 \\
Case 5.2.9 & 0.02857142857142857 & 0.02857142857142857 & 4.341185345099308 & 0.9904731250696808 & 143.5523827878386 \\
Case 5.2.10 & 0.02857142857142857 & 0.02857142857142857 & 4.731222306735877 & 0.9969319295625433 & 506.9119438251631 \\
\hline
\end{tabular}

Table 5 offers seventeen referencing cases. The first ten items are Case 5.3.1 is $q=1, b_{x}=b_{y}=3, \alpha / \beta=1, \alpha^{2}=15$; Case 5.3.2 is $q=1, b_{x}=b_{y}=3, \alpha / \beta=1, \alpha^{2}=1227$; Case 5.3.3 is $q=1, b_{x}=b_{y}=3, \alpha / \beta=1, \alpha^{2}=1228$; Case 5.3.4 is $q=1, b_{x}$ $=b_{y}=3, \alpha / \beta=1, \alpha^{2}=1229$; Case 5.3 .5 is $q=1, b_{x}=b_{y}=3, \alpha$ $/ \beta=1, \alpha^{2}=2900$; Case 5.3.6 is $q=1, b_{x}=b_{y}=3, \alpha / \beta=1, \alpha^{2}$ = 2999; Case 5.3 .7 is $q=1, b_{x}=b_{y}=3, \alpha / \beta=1, \alpha^{2}=3000$; Case 5.3 .8 is $q=1, b_{x}=b_{y}=3, \alpha / \beta=1, \alpha^{2}=8 \times 10^{4}$; Case 
TABLE 5: Quenching data of $\alpha$ and $\beta$ based on convection term $b /(1+\alpha x+\beta y)$.

\begin{tabular}{lccccc}
\hline Case name & $x_{\max }$ & $y_{\max }$ & $T_{\max }$ & $\max$ & $u$ \\
\hline Case 5.3.1 & 0.20 & 0.20 & 3.621624575204209 & 0.991718707508642 & 86.83309533401254 \\
Case 5.3.2 & 0.02857142857142857 & 0.02857142857142857 & 3.609153368630949 & 0.9907185674353032 & 73.0239115821952 \\
Case 5.3.3 & 0.01428571428571429 & 0.01428571428571429 & 3.609390601249229 & 0.9912265622789864 & 150.6673183543721 \\
Case 5.3.4 & 0.01428571428571429 & 0.01428571428571429 & 3.609274948219342 & 0.9919688690187356 & 165.4554013002607 \\
Case 5.3.5 & 0.01428571428571429 & 0.01428571428571429 & 3.376127343223672 & 0.9902039498799047 & 90.20671661807094 \\
Case 5.3.6 & 0.01428571428571429 & 0.01428571428571429 & 3.378639256536471 & 0.9927000295904764 & 120.3354981361145 \\
Case 5.3.7 & 0.01428571428571429 & 0.01428571428571429 & 3.378668197610872 & 0.9923048003313988 & 113.9606848017258 \\
Case 5.3.8 & 0.01428571428571429 & 0.01428571428571429 & 23.38176644203467 & 0.9900781386538231 & 17.60457567575462 \\
Case 5.3.9 & 0.01428571428571429 & 0.01428571428571429 & 31.0931917834407 & 0.9900013538386812 & 14.90527975955028 \\
Case 5.3.10 & 0.01428571428571429 & 0.01428571428571429 & 39.49651984658089 & 0.990003317828528 & 13.07949247781412 \\
\hline
\end{tabular}

TABLE 6: The maximal values and locations of $u$ and $u_{t}$ for $q=1, b_{x}=b_{y}=3, \alpha=\beta=15$ based on convection term $b /(1+\alpha x+\beta y)$.

\begin{tabular}{lcccc}
\hline$t$ & $x$ & $y$ & $\max _{x, y} u$ & $\max _{x, y}\left(u_{t}\right)$ \\
\hline 3.428290495431297 & 0.04285714285714286 & 0.04285714285714286 & 0.983134449815426 & 53.0703838235108 \\
3.428342645817735 & 0.04285714285714286 & 0.04285714285714286 & 0.863372971410449 & 68.08063220416238 \\
3.428379787989620 & 0.04285714285714286 & 0.04285714285714286 & 0.9893206357439858 & 90.30503152559813 \\
3.428404873627137 & 0.04285714285714286 & 0.04285714285714286 & 0.9920620807720361 & 125.6265973017968 \\
3.428420657560073 & - & - & 17.07446290189856 & $1.200141619871376 \times 10^{9}$ \\
\hline
\end{tabular}

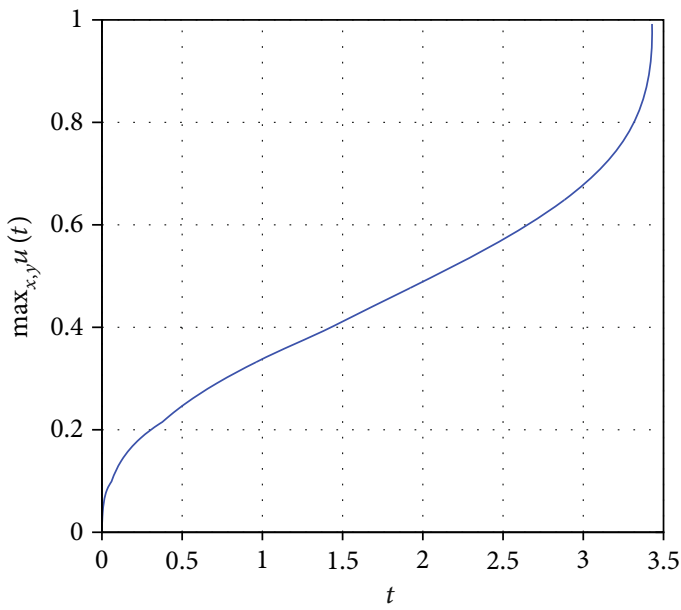

(a)

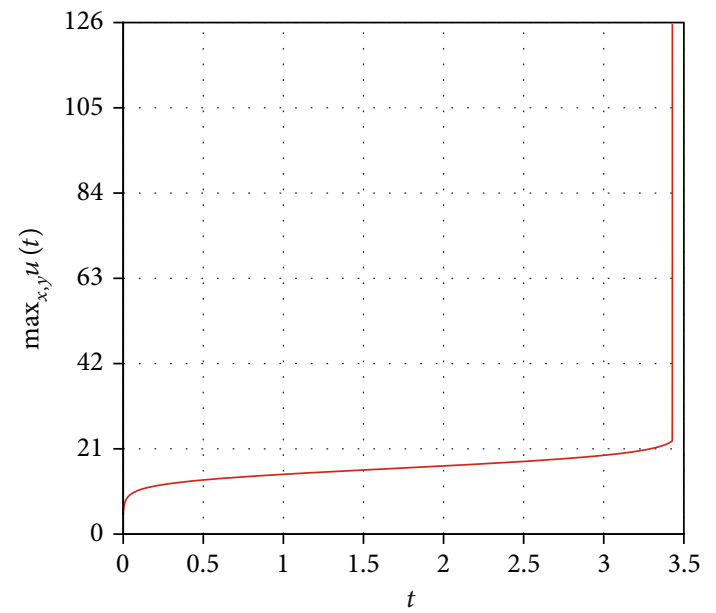

(b)

FIgURE 2: The graphs of solution $u$ and $u_{t}$ : (a) $\max _{x, y} u$ as $t$ increases, and (b) $\max _{x, y}\left(u_{t}\right)$ as $t$ increases from the initial moment to the occurrence of quenching behavior with the parameters $q=1, b_{x}=b_{y}=3, \alpha=\beta=15$ based on convection term $b /(1+\alpha x+\beta y)$.

5.3.9 is $q=1, b_{x}=b_{y}=3, \alpha / \beta=1, \alpha^{2}=1.1 \times 10^{5}$; Case 5.3.10 is $q=1, b_{x}=b_{y}=3, \alpha / \beta=1, \alpha^{2}=1.43 \times 10^{5}$. We configure $15 \leq \alpha^{2} \leq 1.43 \times 10^{5}$ and $\alpha / \beta=1$ for the prior ten items. Experiments show even if $\alpha^{2}$ reaches $10^{5}$, quenching phenomena still happen. Furthermore, if $\alpha^{2}$ falls in the interval $\left[15,1.43 \times 10^{5}\right]$, then quenching phenomena will occur normally, and quenching location $x_{\max }$ and $y_{\max }$ do not decrease monotonously as $\alpha^{2}$ declines. When $\alpha^{2}$ lies in the area of [ $\left.1228,1.43 \times 10^{5}\right], \quad\left(x_{\max }, y_{\max }\right) \quad$ always keeps ( $0.01428571428571429,0.01428571428571429)$. In terms of quenching time, there is no obvious linear relationship between $T_{\max }$ and $\alpha^{2}$ in a small range of the definition field of $\alpha^{2}$. If the measurement scale is enlarged in the domain of $\alpha^{2}$, especially when $\alpha^{2}$ reaches $2900, T_{\max }$ will rise with the increase of $\alpha^{2}$, i.e., $T_{\max }$ is 3.376127343223672 when $\alpha^{2}$ is 2900 and $T_{\max }$ is 39.49651984658089 when $\alpha^{2}$ is $1.43 \times 10^{5}$.

We take Case 5.0 to demonstrate specific quenching phenomena. In this case, quenching occurs at $T_{5} *=$ $3.428404873627137, x_{5} *=0.04285714285714286$, and $y_{5}$ $*=0.04285714285714286$ with the parameters $q=1, b_{x}=$ $b_{y}=3, \alpha=\beta=15$. The next context declares more details about quenching. Let us observe the performances of the 


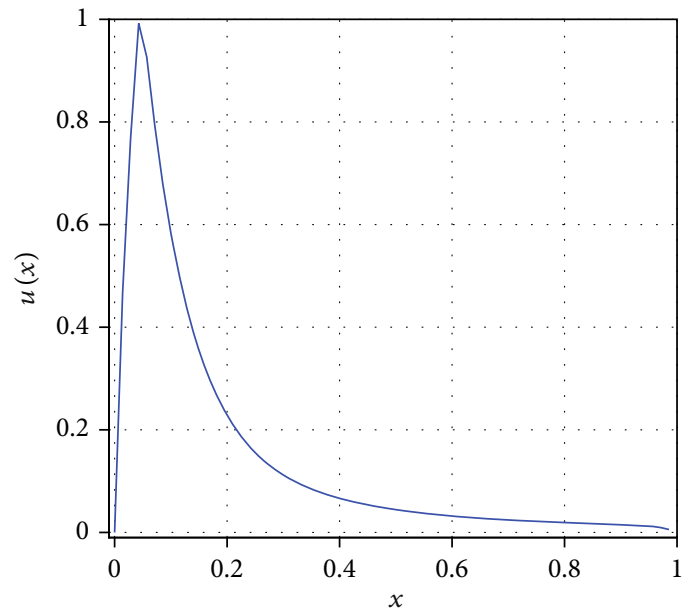

(a)

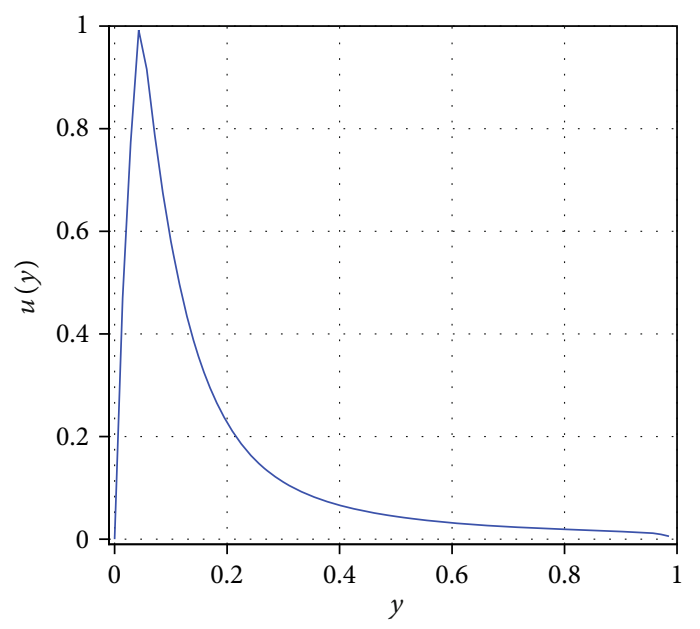

(c)

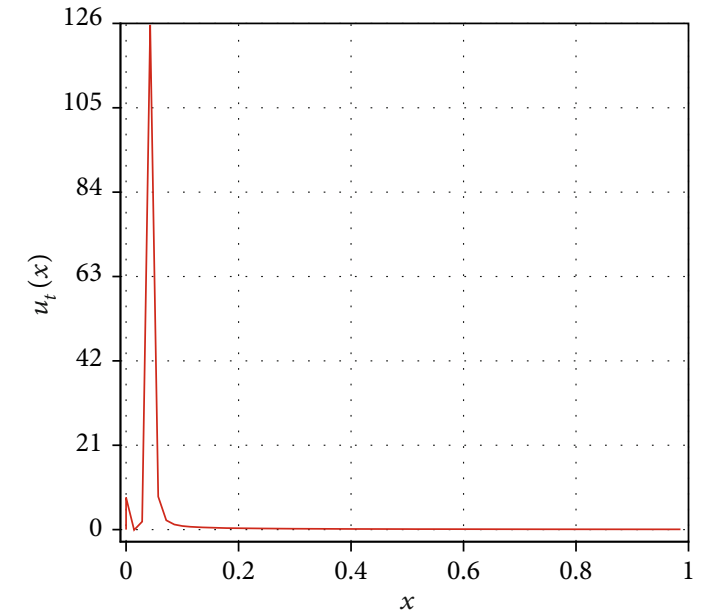

(b)

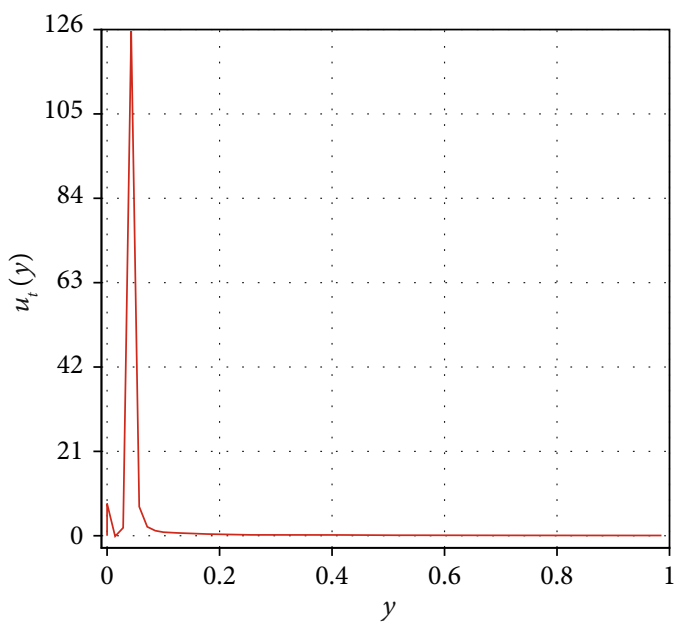

(d)

Figure 3: The graphs of (a) $u$ as $x$ increases, (b) $u_{t}$ as $x$ increases, (c) $u$ as $y$ increases, and (d) $u_{t}$ as $y$ increases when $t$ is $T_{5} *$ for convection term $b /(1+\alpha x+\beta y)$.

solution $u$, the derivative function $u_{t}$, and their time and space locations of five stages relative to quenching for Case 5.0, which show in Table 6 and Figures 2-4. The first four items reflect the four states before quenching occurs in Table 6. The last item represents the quenching state in which $u$ is more than 1 and reaches $17.07446290189856, u_{t}$ blows up and reaches $1.200141619871376 \times 10^{9}$, the position of $\max _{x, y}$ $u$ is $\left(0.5336298640841476 \times 10^{-5}, 0.2902651144597507\right)$, and the position of $\max _{x, y}\left(u_{t}\right)$ is $\left(0.5702149685882448 \times 10^{-4}\right.$, $\left.0.5677032305886261 \times 10^{-4}\right)$ when $t=3.428420657560073$. Figures 2(a) and 2(b) denote the profiles of both solution $u$ and derivative function $u_{t}$. In the aforementioned figures, the maximum values of $u$ and $u_{t}$ grow from 0 to $T_{5} *$. As soon as time variable $t$ arrives at quenching spot $\left(x_{5} * y_{5} *\right)$ and quenching time $T_{5} *$, we have $\max u=0.99206208077203618$ and $\max \left(u_{t}\right)=125.6265973017968$. By locating the quenching time $T_{5} *$, we can draw the plots of $\max _{x, y} u(t)$ and $\max _{x, y} u_{t}(t)$. Figures 3(a)-3(d) depict the function relationships between $u$ and $x$, between $u_{t}$ and $x$, between $u$ and $y$, and between $u_{t}$ and $y$, respectively, when $t$ is $T_{5} *$. Owing to the identity of $x$ and $y$ in the original equation, Figures 3(a) and 3(c) have the same shape, and Figures 3(b) and 3(d) have the same shape.

Figure 4 depicts curve of the adaptive temporal steps as the time variable progresses and graphs of the adaptive spatial steps changing as the space variables change. Figures $4(\mathrm{a})$ and 4(b) portray the distributions of spatial steps. The plots in Figure 4(a) are similar to those in Figure 4(b), but there are some subtle differences in their specific data. The blue curve depicts the tendency of spatial steps varying before quenching occurs, which underlines $x_{5} *\left(y_{5} *\right)$. The green line refers to spatial step distribution of uniform mesh with nonadaption, which is extracted from the initial temporal layer. Mesh adaptation is stimulated at the position of red square sign, and $\tau_{n}$ becomes gradually smaller and falls rapidly toward its floor when $t \longrightarrow T_{5} *$ in Figure 4(c). The red square mark represents the moment $t=3.425399077950409$ and the temporal step $\tau_{n}$ $=5.990779506555022 \times 10^{-4}$ when the process approximates to quenching time. The time adaption is triggered before the moment $T_{5} *$ and lasts through the rest course of calculation. So this quenching phenomenon is caught with the features of $\max _{x, y} u \longrightarrow 1^{-}$and $\max _{x, y}\left(u_{t}\right) \longrightarrow 126^{-}$as $t$ reaches $T_{5} *$. 


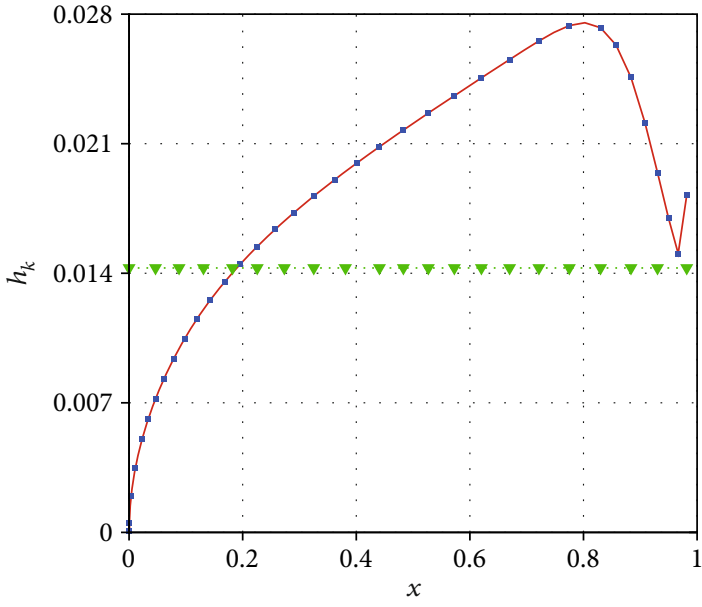

(a)

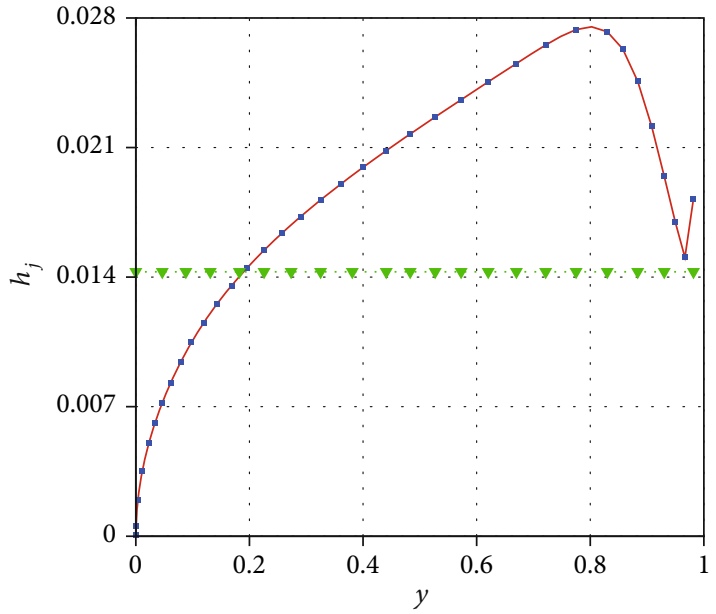

(b)

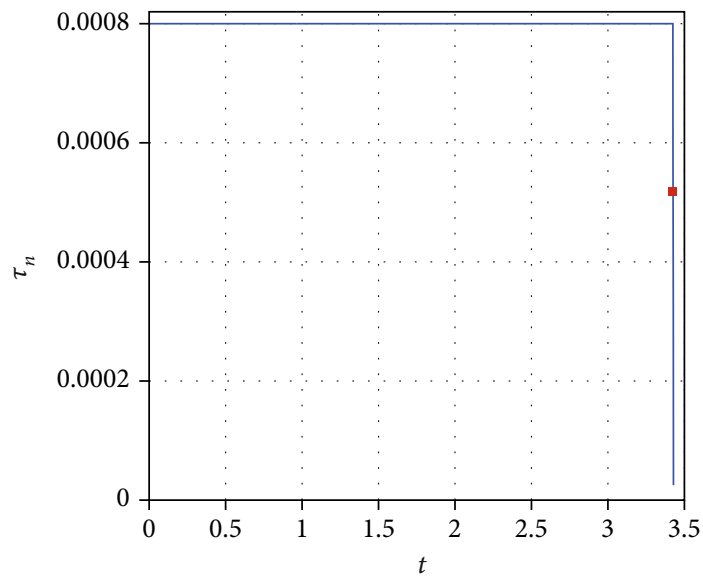

(c)

Figure 4: (a) The variation of spatial step $h_{k}$ as $x$ processes; (b) the variation of spatial step $h_{j}$ as $y$ processes; (c) the curve of the temporal steps $\tau_{n}$ as time $t$ increases with the parameters $q=1, b_{x}=b_{y}=3, \alpha=\beta=15$ based on convection term $b /(1+\alpha x+\beta y)$.

There are three groups of 3D profiles of the solutions and their temporal derivatives in Figure 5. Figures 5(a) and 5(b) denote the two three-dimensional plots of $u$ and $u_{t}$ when $t=$ 0.40 , respectively. Figures $5(\mathrm{c})$ and $5(\mathrm{~d})$ denote the two threedimensional plots of $u$ and $u_{t}$ when $t=3.428342645817735$, respectively. Figures 5(e) and 5(f) denote the two threedimensional plots of $u$ and $u_{t}$ when $t=T_{5} *$, respectively. Thought the 3D plots, we can get richer information. The first group is the three-dimensional views from both $u$ and $u_{t}$ at the first 500th temporal layers in the excursion, in which $\max _{x, y} u$ $\longrightarrow 0.2215$ and $\max _{x, y}\left(u_{t}\right) \longrightarrow 12.9459$ when $t=0.40$. The second group is the three-dimensional views from both $u$ and $u_{t}$ including the penultimate temporal steps before $T_{5} *$, in whichmax $_{x, y} u \longrightarrow 0.9863$ and $\max _{x, y}\left(u_{t}\right) \longrightarrow 68.0806$ when $t=3.428342645817735$. The third group is the threedimensional views from both $u$ and $u_{t}$ immediately before quenching happens, in which $\max _{x, y} u \longrightarrow 0.9921$ and $\max _{x, y}$ $\left(u_{t}\right) \longrightarrow 125.6266$ when $t=3.428404873627137$. During the temporal layers' moving forward, the solution $u$ changes smoothly and then almost arrives at the peak value while $t$ approximates to $T_{5} *$. Its peak is the maximal value but before quenching time and location. There is some subtle perturbation at the initial temporal axis of the left boundary for the change rate of the solution. Afterward, the temporal derivative varies smoothly, and their maximums of each time axis increase steadily. While $t$ approaches to the quenching time $T_{5} *$, the maximum $u_{t}$ also increases rapidly and infinitely and then blows up at the next time layer of quenching time.

4.4. Quenching Example with Convection Term $b /(\alpha x+\beta y)$. Similarly, we need to only consider $\theta=1.0$ and conduct a series of simulation experiments to investigate the $2 \mathrm{D}$ quenching regularity for the convection term $b /(\alpha x+\beta y)$ which is related to the three groups of elements: $q$ and $b_{x}$ and $b_{y}, \alpha$, and $\beta$ and exemplify the quenching behaviors of the representative Case 6.0. The convection term $b /(\alpha x+\beta y$ ) should written as $c_{1}(x, y)=b_{x} /(\alpha x+\beta y)$ and $c_{2}(x, y)=b_{y} /$ $(\alpha x+\beta y)$, where $b_{x}$ and $b_{y}$ take the constants and convection parameter $b=b_{x}=b_{y}$. For Case 6.0, the initial $x$ - step is $h_{0}$ $=1 / 80$, and the initial $t-$ step is $\tau_{0}=0.001$. We choose some representative data to record in Tables 7-10 from the simulation results. Case 6.0 is regarded as the reference standard 


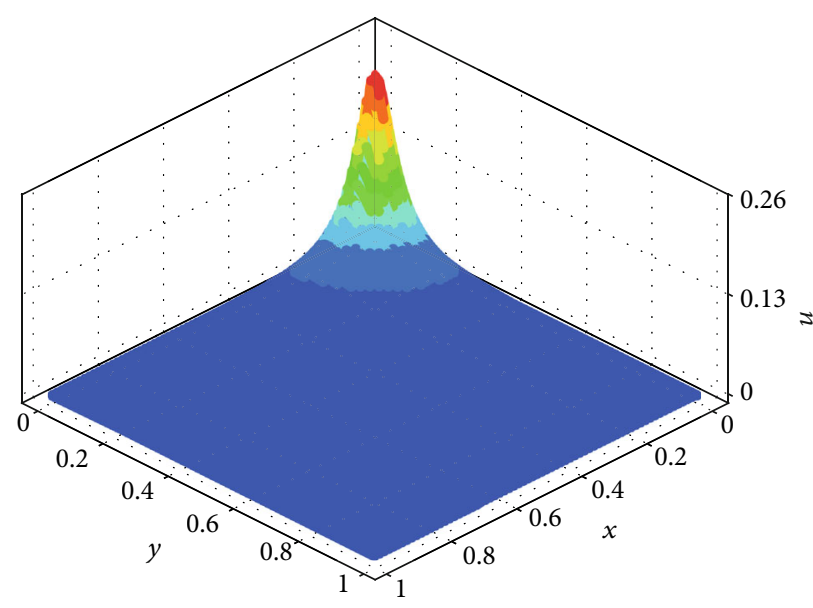

(a)

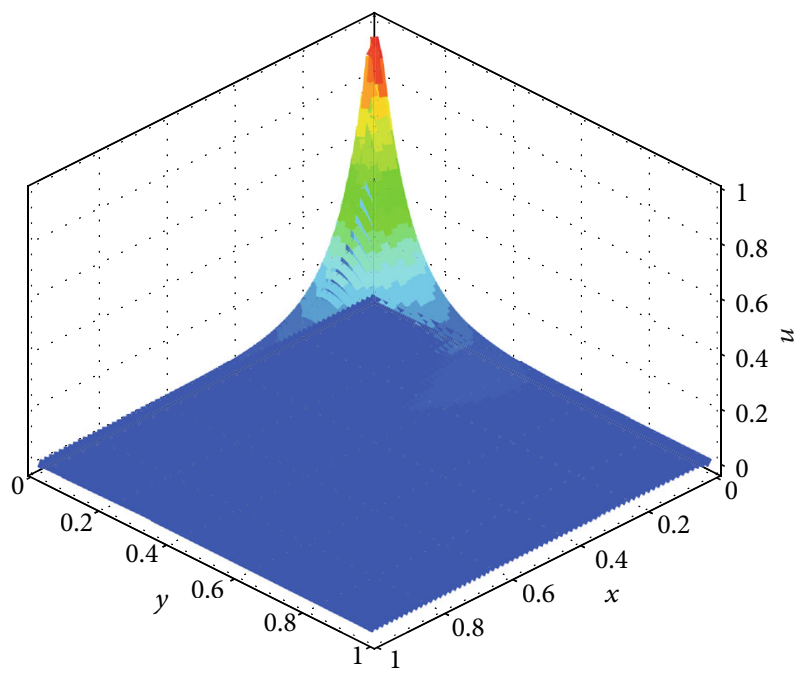

(c)

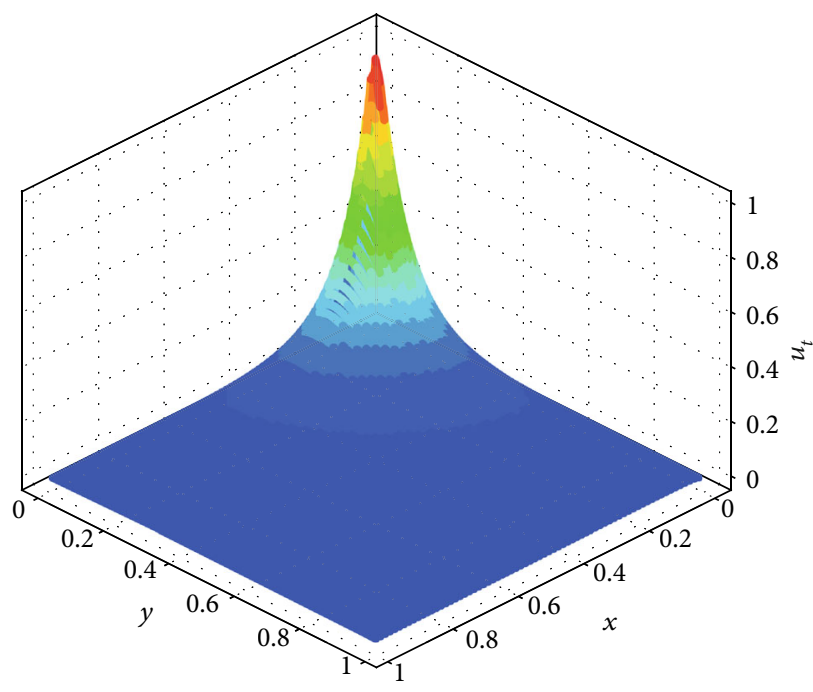

(e)

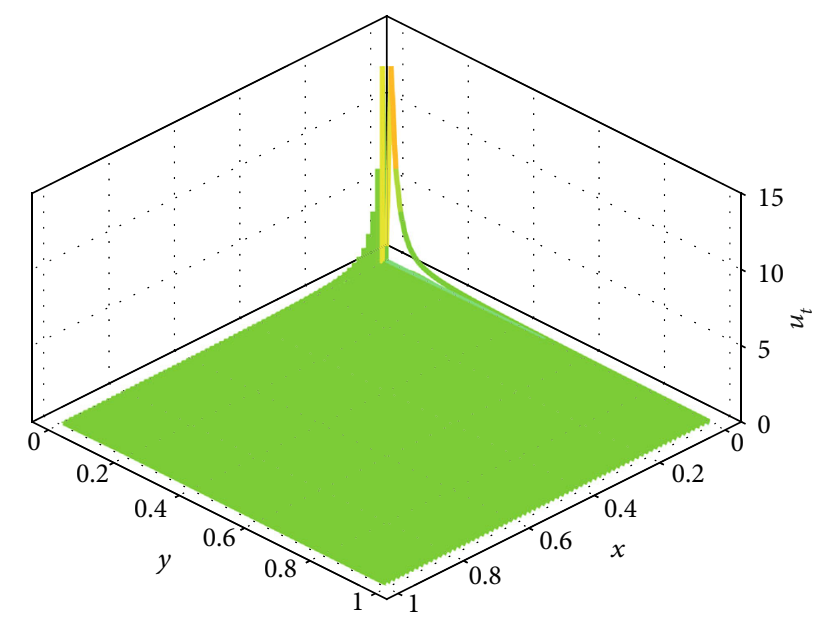

(b)

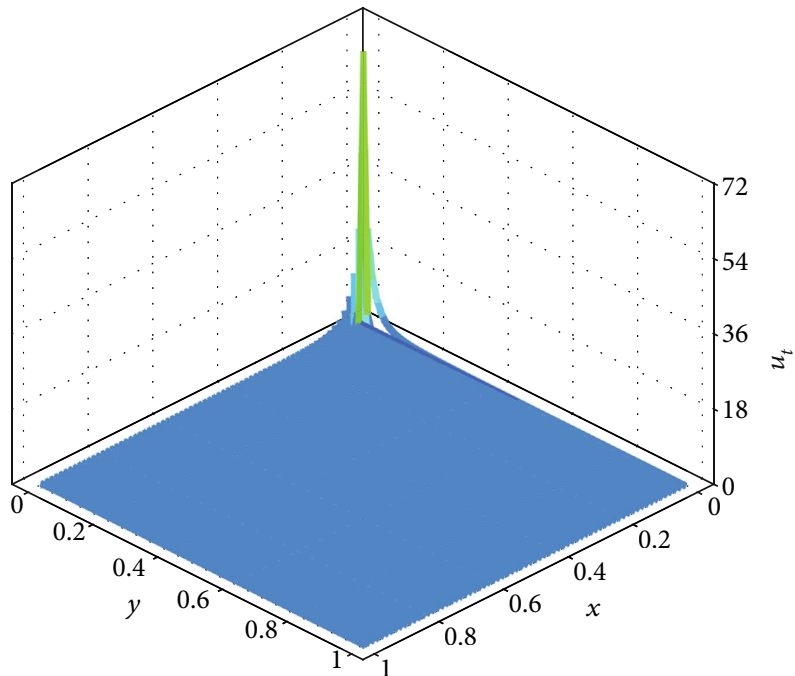

(d)

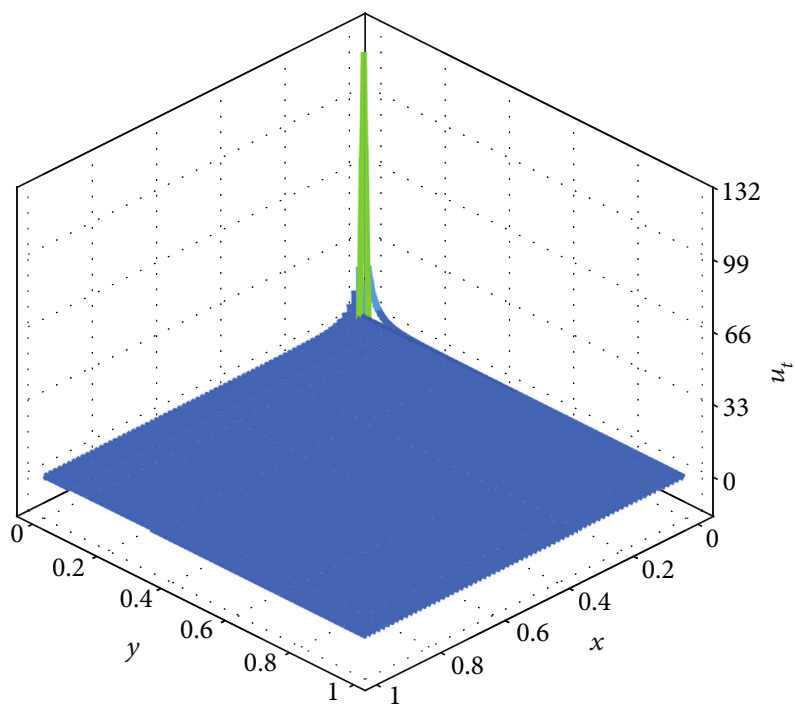

(f)

Figure 5: (a) The 3D plot of $u$ when $t=0.40$; (b) the 3D plot of $u_{t}$ when $t=0.40$; (c) the 3D plot of $u$ when $t=3.428342645817735$; (d) the 3D plot of $u_{t}$ when $t=3.428342645817735$; (e) the 3D plot of $u$ when $t=T_{5} *$; (f) the 3D plot of $u_{t}$ when $t=T_{5} *$. All is for Case 5.0. 
TABLE 7: Quenching data of $q$ based on convection term $b /(\alpha x+\beta y)$.

\begin{tabular}{lccccc}
\hline Case name & $x_{\max }$ & $y_{\max }$ & $T_{\max }$ & $\max u$ & $\max u_{t}$ \\
\hline Case 6.0 & 0.0125 & 0.0125 & 4.020449234557191 & 0.981186659892471 & 31.34049546140411 \\
Case 6.1.1 & 0.025 & 0.0375 & 0.4893400471790108 & 0.9815547233986595 & 53.96815295250957 \\
Case 6.1.2 & 0.025 & 0.025 & 1.40546552826671 & 0.9810673165994175 & 30.97740120847389 \\
Case 6.1.3 & 0.025 & 0.0125 & 1.806856006612364 & 0.9835285046535343 & 34.84710326333095 \\
Case 6.1.4 & 0.0125 & 0.0125 & 2.315800912037878 & 0.9860032076007205 & 48.09280080596852 \\
Case 6.1.5 & 0.0125 & 0.0125 & 3.004283302198541 & 0.9847606913347033 & 41.42159866823076 \\
Case 6.1.6 & 0.025 & 0.025 & 5.519520896140588 & 0.9804916164771977 & 15.56936805077945 \\
Case 6.1.7 & 0.025 & 0.025 & 7.6129781181848 & 0.9806093675789447 & 13.71511944823688 \\
Case 6.1.8 & 0.025 & 0.025 & 10.86315690956873 & 0.9803668713972701 & 11.86327696425435 \\
Case 6.1.9 & 0.025 & 0.025 & 16.29059159714386 & 0.9800718066608986 & 10.23980977162735 \\
Case 6.1.10 & 0.025 & 0.025 & 26.33869553245833 & 0.9802129067546791 & 9.042418195146764 \\
Case 6.1.11 & 0.025 & 0.025 & 47.98116891173758 & 0.980293256909013 & 7.964013508962916 \\
\hline
\end{tabular}

TABLE 8: Quenching data of $b_{x}$ and $b_{y}$ based on convection term $b /(\alpha x+\beta y)$.

\begin{tabular}{lccccc}
\hline Case name & $x_{\max }$ & $y_{\max }$ & $T_{\max }$ & $\max u$ & $\max u_{t}$ \\
\hline Case 6.2.1 & 0.0625 & 0.0625 & 10.99093267786784 & 0.9804498472291583 & 8.583794969514605 \\
Case 6.2.2 & 0.0375 & 0.0375 & 7.809655701953323 & 0.9804503193889351 & 12.7219405872281 \\
Case 6.2.3 & 0.0375 & 0.0375 & 6.210534222541208 & 0.9809122863640634 & 13.14389285559599 \\
Case 6.2.4 & 0.025 & 0.025 & 4.956004105630788 & 0.9818372265165442 & 18.9029625958216 \\
Case 6.2.5 & 0.0125 & 0.0125 & 2.64799664630179 & 0.9810960736197033 & 31.29445994657764 \\
Case 6.2.6 & 0.0125 & 0.0125 & 9.559656653594685 & 0.9813004101488958 & 33.42529207679014 \\
Case 6.2.7 & 0.025 & 0.025 & 18.1622615973841 & 0.9808985418280476 & 18.03120629119135 \\
Case 6.2.8 & 0.0375 & 0.025 & 38.77536139517031 & 0.9804090344629824 & 14.57573131335622 \\
Case 6.2.9 & 0.0375 & 0.0375 & 59.07244177030795 & 0.9802503334133788 & 12.64186937140285 \\
Case 6.2.10 & 0.0375 & 0.0375 & 89.05678402848356 & 0.9803538247671133 & 13.00866087970327 \\
\hline
\end{tabular}

TABLE 9: Quenching data of $\alpha$ and $\beta$ based on convection term $b /(\alpha x+\beta y)$.

\begin{tabular}{lccccc}
\hline Case name & $x_{\max }$ & $y_{\max }$ & $T_{\max }$ & $\max u$ & $\max u_{t}$ \\
\hline Case 6.3.1 & 0.5375 & 0.5375 & 8.240022739766165 & 0.9805231629517244 & 19.33141003501417 \\
Case 6.3.2 & 0.1229 & 0.1229 & 6.240792928817466 & 0.9804429759036448 & 21.02610806687704 \\
Case 6.3.3 & 0.0625 & 0.0625 & 3.431334351295505 & 0.9809418101698555 & 21.77295829105509 \\
Case 6.3.4 & 0.0375 & 0.0375 & 3.441737958890371 & 0.9816372001957696 & 23.0950930660414 \\
Case 6.3.5 & 0.025 & 0.025 & 3.750551299221261 & 0.9801324957943957 & 19.04800266218939 \\
Case 6.3.6 & 0.025 & 0.025 & 4.024152273164159 & 0.9800420381170325 & 17.6646128655794 \\
Case 6.3.7 & 0.025 & 0.025 & 4.065155138423362 & 0.9805944201781043 & 18.01149509967014 \\
Case 6.3.8 & 0.0125 & 0.0125 & 4.06558591278706 & 0.9807065134704962 & 30.77480165240926 \\
Case 6.3.9 & 0.0125 & 0.0125 & 3.520143605280937 & 0.9809208903166006 & 24.6618068728011 \\
Case 6.3.10 & 0.0125 & 0.0125 & 13.44243402330071 & 0.9805575860550446 & 8.352004051122194 \\
\hline
\end{tabular}

and defined as $q=0.8, b_{x}=b_{y}=-2, \alpha=\beta=\sqrt{11000}$. Tables 7-10 and Figures 6-9 demonstrate specific quenching information of Case 6.0.

The effect of $q$ on quenching problem can be illustrated by continual experiments. A reasonable quenching behavior will occur when $q=0$ or $q$ takes a value in $[0.4,1.4]$. When $q$ is $0.1,0.2,0.3$, or $q \geq 1.5$, it does not produce quenching phenomena. There are quenching characteristics for $q$ : quenching location $x_{\max }$ is not equal to $y_{\max }$ when $q$ takes $0,0.5$ while $x_{\max }$ is equal to $y_{\max }$ in the other cases; ( $x_{\max }$, $\left.y_{\max }\right)$ is $(0.0125,0.0125)$ when $q$ takes $0.6,0.7$, and 0.8 ; $\left.x_{\max }, y_{\max }\right)$ is $(0.025,0.025)$ when $q$ is in $[0.9,1.4]$. Quenching time $T_{\max }$ increases as $q$ increases in the domain of $q$. Table 7 gives some of these quenching cases, in which Case 6.1.1 is $q=0, b_{x}=b_{y}=-2, \alpha=\beta=\sqrt{11000}$; Case 6.1.2 is $q=$ $0.4, b_{x}=b_{y}=-2, \alpha=\beta=\sqrt{11000}$; Case 6.1.3 is $q=0.5, b_{x}=$ 
TABLE 10: The maximal values and locations of $u$ and $u_{t}$ for $q=0.8, b_{x}=b_{y}=-2, \alpha=\beta=\sqrt{11000}$ based on convection term $b /(\alpha x+\beta y)$.

\begin{tabular}{lcccc}
\hline$t$ & $x$ & $y$ & $\max _{x, y} u$ & $\max _{x, y}\left(u_{t}\right)$ \\
\hline 3.946999999999676 & 0.0125 & 0.0125 & 0.8195986394102188 & 2.499122191096586 \\
3.999999999999671 & 0.0125 & 0.0125 & 0.8921719731921093 & 4.684299269080528 \\
4.016999999999676 & 0.0125 & 0.0125 & 0.9488686925507734 & 10.88364621771482 \\
4.019595682766669 & 0.0125 & 0.0125 & 0.9692844094996356 & 18.7880259490986 \\
4.020449234557191 & 0.0125 & 0.0125 & 0.981186659892471 & 31.34049546140411 \\
4.02068525061823 & - & - & $5.358959032595640 \times 10^{16}$ & $7.966445413263532 \times 10^{19}$ \\
\hline
\end{tabular}

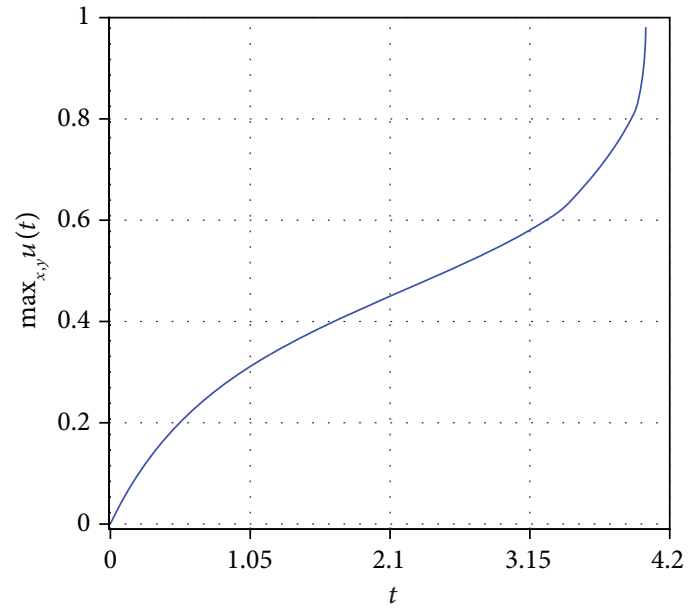

(a)

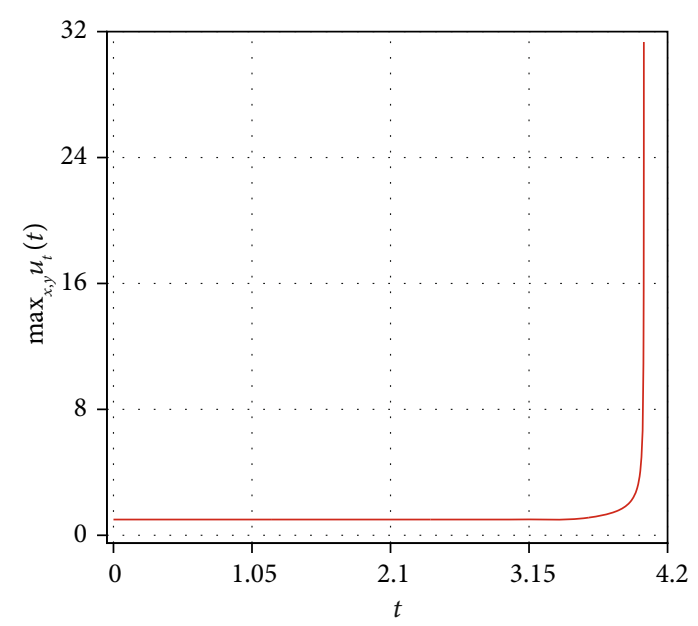

(b)

FIGURE 6: The graphs of solution $u$ and $u_{t}$ : (a) $\max _{x, y} u$ as $t$ increases, and (b) $\max _{x, y}\left(u_{t}\right)$ as $t$ increases from the initial time to the occurrence of quench behavior with $q=0.8, b_{x}=b_{y}=-2, \alpha=\beta=\sqrt{11000}$ based on convection term $b /(\alpha x+\beta y)$.

$b_{y}=-2, \alpha=\beta=\sqrt{11000}$; Case 6.1 .4 is $q=0.6, b_{x}=b_{y}=-2$, $\alpha=\beta=\sqrt{11000}$; Case 6.1 .5 is $q=0.7, b_{x}=b_{y}=-2, \alpha=\beta=$ $\sqrt{11000}$; Case 6.1.6 is $q=0.9, b_{x}=b_{y}=-2, \alpha=\beta=\sqrt{11000}$; Case 6.1.7 is $q=1, b_{x}=b_{y}=-2, \alpha=\beta=\sqrt{11000}$; Case 6.1.8 is $q=1.1, b_{x}=b_{y}=-2, \alpha=\beta=\sqrt{11000}$; Case 6.1.9 is $q=1.2$ , $b_{x}=b_{y}=-2, \alpha=\beta=\sqrt{11000}$; Case 6.1 .10 is $q=1.3, b_{x}=b_{y}$ $=-2, \alpha=\beta=\sqrt{11000}$; Case 6.1 .11 is $q=1.4, b_{x}=b_{y}=-2, \alpha$ $=\beta=\sqrt{11000}$; Case 6.0 is between Case 6.1.5 and Case 6.1.6.

The paragraphs describe the relationship between $b_{x}\left(b_{y}\right)$ and quenching features. We write ten cases in Table 8. Case 6.2.1 is $q=0.8, b_{x}=b_{y}=-16, \alpha=\beta=\sqrt{11000}$; Case 6.2.2 is $q=0.8, b_{x}=b_{y}=-10, \alpha=\beta=\sqrt{11000}$; Case 6.2.3 is $q=0.8$, $b_{x}=b_{y}=-7, \alpha=\beta=\sqrt{11000}$; Case 6.2.4 is $q=0.8, b_{x}=b_{y}=$ $-5, \alpha=\beta=\sqrt{11000}$; Case 6.2 .5 is $q=0.8, b_{x}=b_{y}=1, \alpha=\beta$ $=\sqrt{11000} ;$ Case 6.2 .6 is $q=0.8, b_{x}=b_{y}=12, \alpha=\beta=$ $\sqrt{11000}$; Case 6.2 .7 is $q=0.8, b_{x}=b_{y}=21, \alpha=\beta=\sqrt{11000}$; Case 6.2 .8 is $q=0.8, b_{x}=b_{y}=40, \alpha=\beta=\sqrt{11000}$; Case 6.2 .9 is $q=0.8, b_{x}=b_{y}=57, \alpha=\beta=\sqrt{11000}$; Case 6.2 .10 is $q=0.8, b_{x}=b_{y}=80, \alpha=\beta=\sqrt{11000}$; Case 6.0 is between Case 6.2.4 and Case 6.2.5. Quenching behavior can be formed when $b_{x}\left(b_{y}\right)$ takes the values in $[-16,80]$, in which the function curve of $\left(b, T_{\max }\right)$ is concave and $T_{\max }$ is smallest when $b=1$. When $b=-16, b=-13, b \in[-10,-7], b \in[-5,12]$, $b \in[21,39]$, or $b \in[57,80]$, quenching location $x_{\max }$ is equal to $y_{\max }$ whereas when $b$ takes other values in $[-16,80], x_{\max }$ is different from $y_{\max }$.

We describe the influence of $\alpha$ and $\beta$ on quenching results just depending on quenching data in Table 9. Of course, we do a great quantity of experiments, from which some typical cases chosen in Table 9. We set $\alpha / \beta=1$ and $\alpha^{2}$ from 10 to $3.0 \times 10^{6}$ for Cases 6.3.1-6.3.10. Specifically, Case 6.3.1 is $q=0.8, b_{x}=$ $b_{y}=-2, \alpha / \beta=1, \alpha^{2}=10$; Case 6.3.2 is $q=0.8, b_{x}=b_{y}=-2, \alpha$ $/ \beta=1, \alpha^{2}=231$; Case 6.3 .3 is $q=0.8, b_{x}=b_{y}=-2, \alpha / \beta=1$, $\alpha^{2}=1000$; Case 6.3 .4 is $q=0.8, b_{x}=b_{y}=-2, \alpha / \beta=1, \alpha^{2}=$ 2756; Case 6.3 .5 is $q=0.8, b_{x}=b_{y}=-2, \alpha / \beta=1, \alpha^{2}=8648$; Case 6.3.6 is $q=0.8, b_{x}=b_{y}=-2, \alpha / \beta=1, \alpha^{2}=10500$; Case 6.3.7 is $q=0.8, b_{x}=b_{y}=-2, \alpha / \beta=1, \alpha^{2}=10775$; Case 6.3.8 is $q=0.8, b_{x}=b_{y}=-2, \alpha / \beta=1, \alpha^{2}=10779$; Case 6.3.9 is $q=$ $0.8, b_{x}=b_{y}=-2, \alpha / \beta=1, \alpha^{2}=19991$; Case 6.3 .10 is $q=0.8$, $b_{x}=b_{y}=-2, \alpha / \beta=1, \alpha^{2}=300000$; Case 6.0 is between Case 6.3.8 and Case 6.3.9.

It does not form quenching status when $\alpha^{2}<10$ or $\alpha^{2}$ $<3.0 \times 10^{6}$. After $\alpha / \beta$ is defined as $1, \alpha^{2}$ is evaluated from 10 to $3.0 \times 10^{6}$. We find that it is more likely to produce quenching behaviors when $\alpha^{2}$ is between 10 and $3.0 \times 10^{6}$. By means of continual tests, Cases 6.3.1-6.3.10 represent 


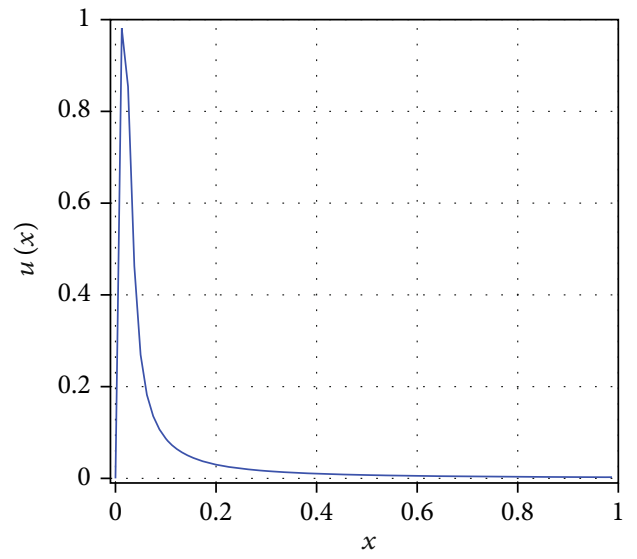

(a)

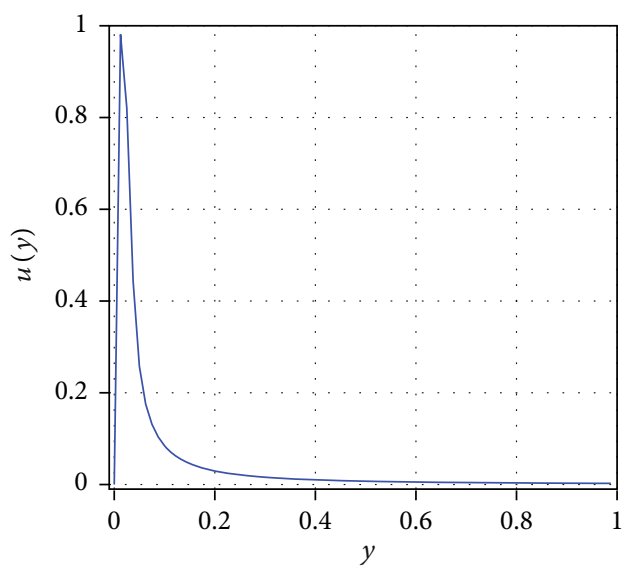

(c)

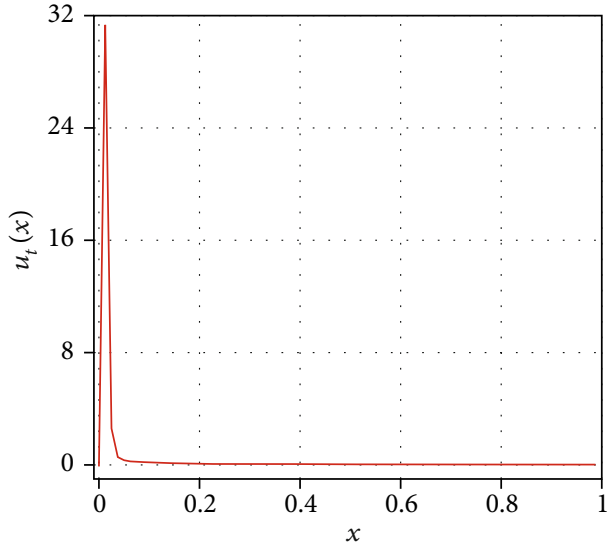

(b)

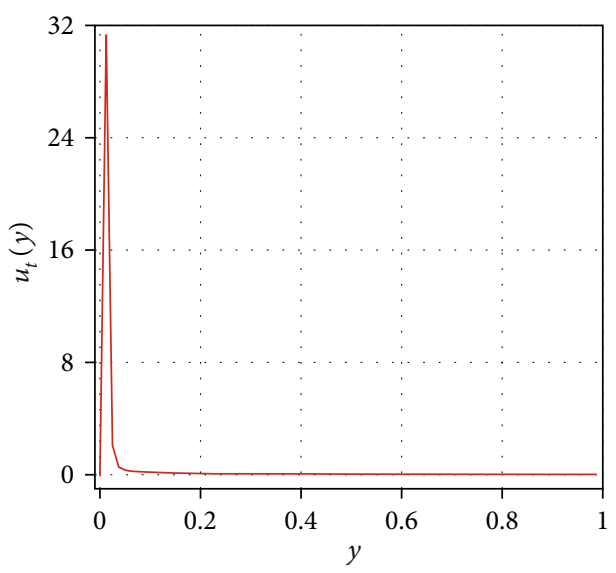

(d)

Figure 7: The graphs of (a) $u$ as $x$ increases, (b) $u_{t}$ as $x$ increases, (c) $u$ as $y$ increases, and (d) $u_{t}$ as $y$ increases when $t$ is $T_{5} *$ for convection term $b /(1+\alpha x+\beta y)$.

the ten special cases, and we can observe the quenching status as follows. As far as quenching spatial characteristic is concerned, quenching location does not monotonously increase as $\alpha^{2}$ increases when $\alpha^{2}$ is in the definition domain. For example, quenching location reaches the maximum $(0.5375$ , 0.5375) when $\alpha^{2}=10$ and quenching location keeps the coordination point of $(0.0125,0.0125)$ when $\alpha^{2}$ is in $[10780$ , $\left.3.0 \times 10^{6}\right]$. Next, quenching temporal characteristic is concerned. In fact, there does not exist strict linear relationship between quenching time and $\alpha^{2}$. Comparatively, quenching time is smaller in the former half than in the latter half of the domain of $\alpha^{2}$. When the measurement scale is enlarged in the domain of $\alpha^{2}$, especially when $\alpha^{2}$ reaches 19991, $T_{\max }$ increases with the increase of $\alpha^{2}$.

Quenching phenomena of Case 6.0 can be considered in the following text. Its quenching time appears at $T_{6} *=$ 4.020449234557191, and its quenching position appears at ( $\left.x_{6} * y_{6} *\right)=(0.0125,0.0125)$ in this situation. Table 10 shows six stages around quenching for Case 6.0. The items from the first to the fifth describe the five stages of representation before the occurrence of quenching, and the last item just notes the quenching moment. $\max _{x, y} u$ is $5.358959032595640 \times 10^{16}$ at $\left(2.0588245639243 \times 10^{-4}, 9.654095827421034 \times 10^{-1}\right)$, and $\max _{x, y}\left(u_{t}\right) \quad$ is $\quad 7.966445413263532 \times 10^{19} \quad$ at $\left(2.080224198836234 \times 10^{-5}, \quad 9.824584434865121 \times 10^{-1}\right)$ when quenching occurs. A comprehensive statement of quenching states with the parameters $q=0.8, b_{x}=b_{y}=-2, \alpha$ $=\beta=\sqrt{11000}$ is recorded in the next paragraphs. Although there is slight perturbation in the left boundary of $u_{t}$, it does not influences on distribution of $u_{t}$. It is evident that Figures 6(a) and 6(b) give two pairs curves of both $\max _{x, y} u$ and $\max _{x, y}\left(u_{t}\right)$. The first one is for the distribution of the solution $\max _{x, y} u(t)$ as $t$ varies, and the second one is for the distribution of $\max _{x, y} u_{t}(t)$ as $t$ increases. There is small perturbation near the start-up of the adaptive procedure for the left figure of $\max _{x, y}\left(u_{t}\right)$. The four figures in Figure 7 give function relationship physical quantities between $u, u_{t}$, and spatial variables. Figure $7(\mathrm{a})$ paints the contour of $(x, u)$, Figure $7(\mathrm{~b})$ paints the contour of $\left(x, u_{t}\right)$, Figure $7(\mathrm{c})$ paints the contour of $(y, u)$, and Figure $7(\mathrm{~d})$ paints the contour of $(y$ ,$\left.u_{t}\right)$. Similarly, Figures $7(\mathrm{a})$ and $7(\mathrm{c})$ have the same shape, and Figures $7(\mathrm{~b})$ and $7(\mathrm{~d})$ have the same shape.

There are three graphics in Figure 8, which represent three function relationships between spatial step in $x-$ direction and $x$, between spatial step in $y$-direction and $y$, 


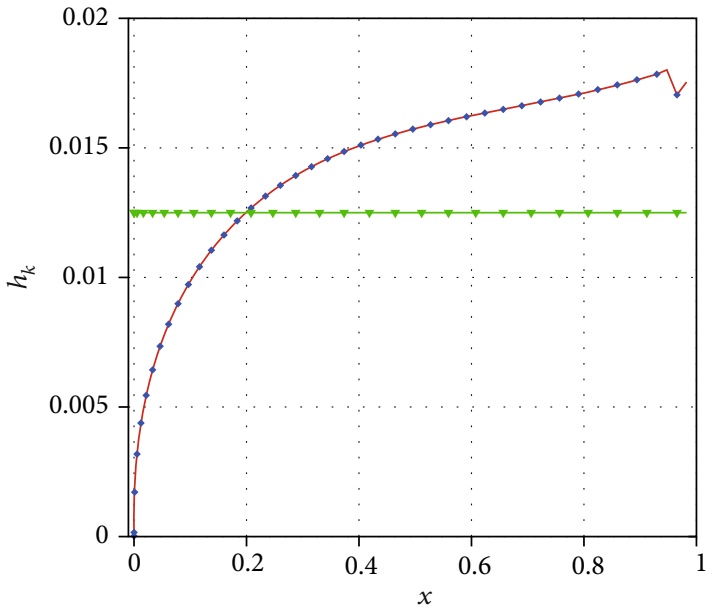

(a)

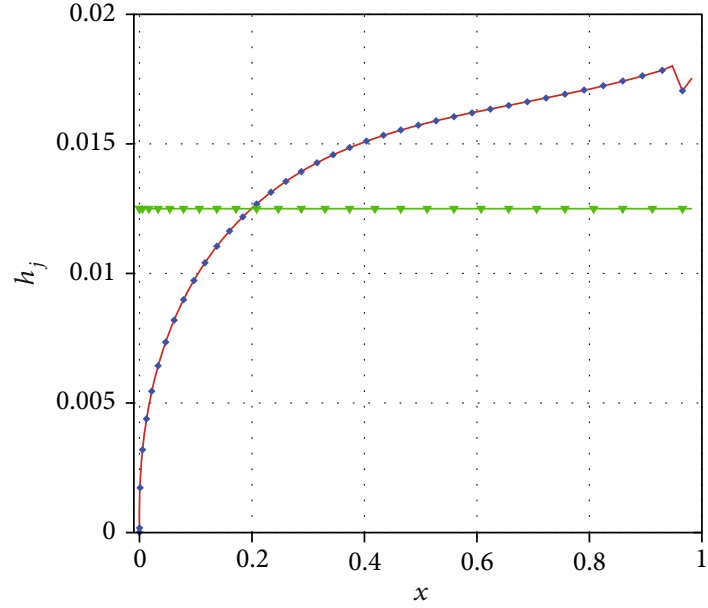

(b)

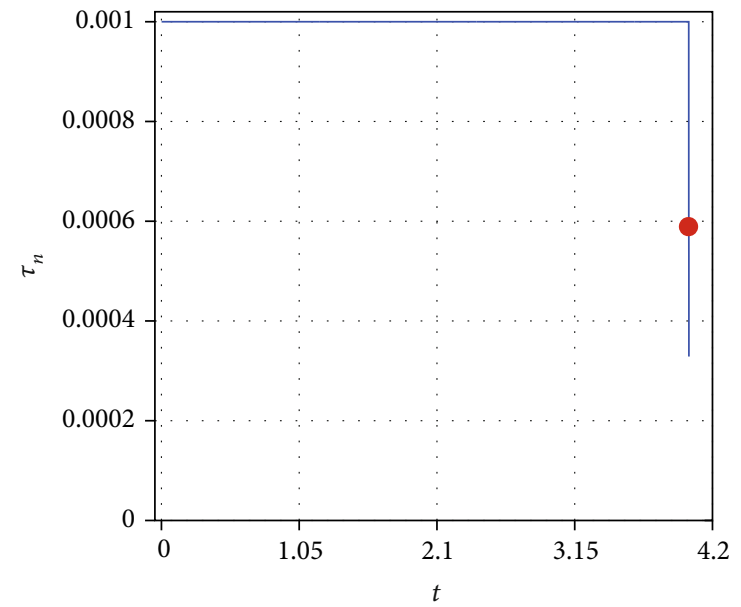

(c)

Figure 8: (a) The variation of spatial step $h_{k}$ as space $x$ processes; (b) the variation of spatial step $h_{j}$ as space $y$ processes; (c) the curve of the temporal steps $\tau_{n}$ as time $t$ increases with the parameters $q=0.8, b_{x}=b_{y}=-2, \alpha=\beta=\sqrt{11000}$ based on convection term $b /(\alpha x+\beta y)$.

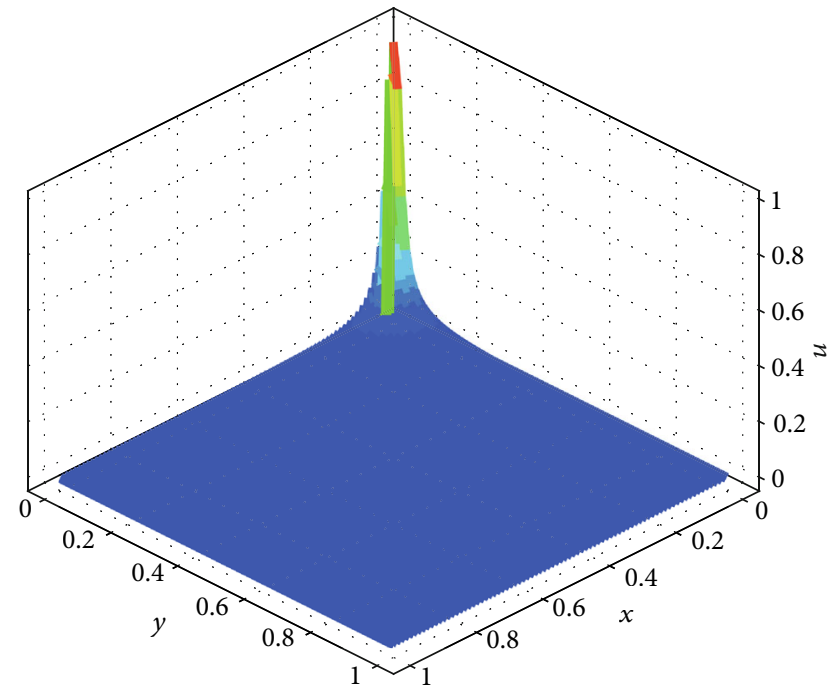

(a)

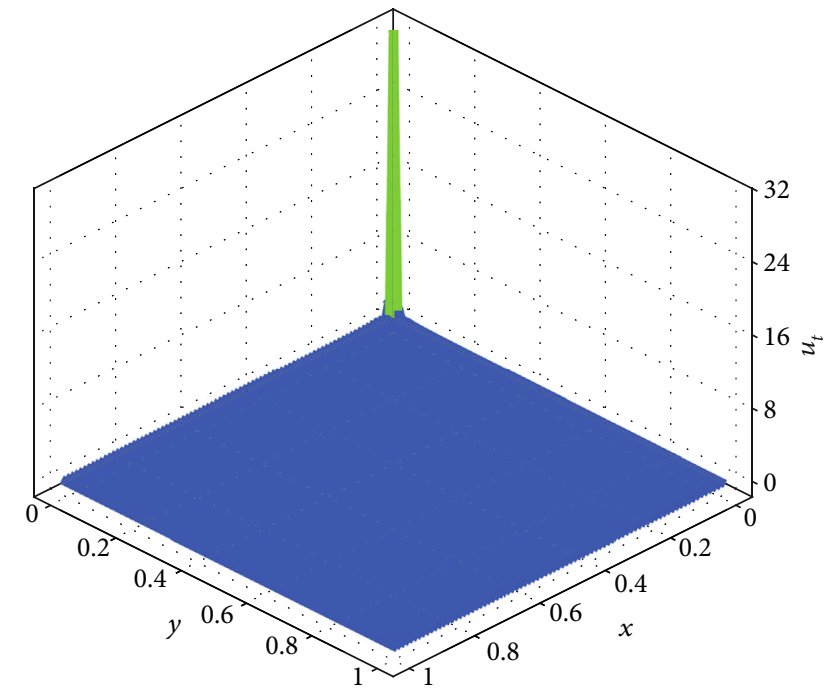

(b)

Figure 9: (a) The 3D plot of $u$ when $t=T_{6} *$ and (b) the 3D plot of $u_{t}$ when $t=T_{6} *$ for Case 6.0. 
and between temporal step and $t$, respectively. Figure 8(a) depicts two curves, in which one is between initial spatial steps $h_{k}$ and $x$, and another is between quenching spatial steps $h_{k}$ and $x$. Figure 8(b) also describes two curves, in which one is between initial spatial steps $h_{k}$ and $y$, and another is between quenching spatial steps $h_{k}$ and $y$. The green lines with gradient marks reflect the initial spatial step distribution that is uniform regardless of $x$-direction or $y$-direction. The red curves covered by blue squares reflect quenching spatial steps that are self-adaptive when quenching occurs. There is a blue curve with a red square marks in Figure 8(c), which portrays temporal step $\tau_{n}$ varies as $t$ changes from 0 to $T_{6} *$. $\tau_{n}$ keeps 0.001 when $0 \leq t<$ 4.019595682766669, but $\operatorname{after} \tau_{n}$ becomes $5.956827669919562 \times 10^{-4}$ when $t=4.019595682766669$ which signed as a red square, $\tau_{n}$ sharply declines up to $3.286760944343214 \times 10^{-4}$ when $t=T_{6} *$.

The three-dimensional surfaces can render more reliable information for both the solution $u$ and the time derivative $u_{t}$ which display in Figure 9. The group of surfaces is the threedimensional views of both $u$ and $u_{t}$ when $t$ is equal to $T_{6} *$. It is smooth and steady that the solution $u$ carries forward at the temporal axis in Figure 9(a). Moreover, it is almost at the middle position of each time axis that the case gives birth to the peak value of $u$. When the position $\left(x_{6} *, y_{6} *\right)$ is at ( $0.0125,0.0125)$ immediately before quenching occurs, the peak value of the solution approaches 1 . The threedimensional representation of Figure 9(b) reveals the evolution of the temporal derivative with regard to spatial variables. The temporal derivative of the solution varies smoothly and reaches its maximums at the quenching domain $(0.0125,0.0125)$. At last, the temporal derivative becomes infinite when quenching occurs at another location $\left(2.080224198836234 \times 10^{-5}, \quad 9.824584434865121 \times 10^{-1}\right)$, which is not observed in Figure 9(b). It is obvious for Case 6.0 that quenching occurs based on $\max _{x, y} u \longrightarrow 1^{-}$and $\max _{x, y}\left(u_{t}\right) \longrightarrow 32^{-}$as $t$ reaches $T_{6} *$.

\section{Conclusion}

Relying on the analyses in this paper, we can sort up the relationship between the parameters $q, b_{x}\left(b_{y}\right), a(\beta)$, and quenching behaviors. There exist four aspects concluded as follows. First, the degenerate function acts within the normal range if the degeneracy parameter $q$ is larger than or equal to 0.4 but not more than 1.3, and quenching time increases as $q$ increases. Second, there is a special point $b^{\wedge}$ dividing the definition domain of convection parameter $b$ as the left and the right subdomain, in which quenching phenomena occur normally and have a variety of features. Quenching time decreases as $b$ increases in the left subdomain, and quenching time increases as $b$ increases in the right subdomain. Quenching location either decreases or convergences upon a certain value as $b$ increases in the domain of $b$. Third, under the condition of $\alpha / \beta=1$, the influence of $\alpha^{2}$ on quenching result is researched. There exists still a special point $\alpha^{\wedge}$ categorizing the definition domain of $\alpha^{2}$ as two subdomains, in which quenching phenomena occur normally and display different spatial effects. Specially, with the rise of $\alpha^{2}$, quenching location does not increases monotonously in the left hand of $\alpha^{\wedge}$ and tends to a fixed coordinates in the right hand of $\alpha^{\wedge}$. Additionally, if we rely on a small scale to observe the domain of $\alpha^{2}$, then there is no linear relationship between quenching time and $\alpha^{2}$. We investigate the definition by virtue of a big scale to find that quenching time also increases when $\alpha^{2}$ become larger, especially when $\alpha^{2}$ reaches certain value; quenching time increases as $\alpha^{2}$ rises. Fourth, it must be a good choice to set $\theta$ as 1 . Through experiments, it is hard to produce quenching phenomena that can be formed when $\theta \geq 2$. From the result analyses, it can be discovered that it is meaningful to initially investigate the $2 \mathrm{D}$ singularity degeneracy problem of quenching type based on the unsteady convection-reaction-diffusion equation by using the highorder difference scheme, which will probably lead to the potentially support to research the next quenching problems.

\section{Data Availability}

The data used to support the findings of this study are available from the corresponding author upon request.

\section{Conflicts of Interest}

The authors declare that they have no conflicts of interest.

\section{Acknowledgments}

This work is partially supported by the National Natural Science Foundation of China (Grant nos. 11772165, 11961054), the National Natural Science Foundation of Ningxia (Grant no. 2018AAC02003), the Key Research and Development Program of Ningxia (Grant no. 2018BEE03007), and the Major Innovation Projects for Building First-class Universities in China's Western Region (Grant no. ZKZD2017009).

\section{References}

[1] K. S. Ha, R. J. Park, S. B. Kim, and H. D. Kim, "Experimental investigations of the quenching phenomena for hemispherical downward facing convex surfaces with narrow gaps," International Communications in Heat and Mass Transfer, vol. 34, no. 1, pp. 28-36, 2007.

[2] J. Lee, G. Son, and H. Y. Yoon, "Numerical simulation of the quenching process in liquid jet impingement," International Communications in Heat and Mass Transfer, vol. 61, pp. 146-152, 2015.

[3] H. Ramezanzadeh, A. Ramiar, and M. Yousefifard, "Numerical investigation into coolant liquid velocity effect on forced convection quenching process," Applied Thermal Engineering, vol. 122, pp. 253-267, 2017.

[4] J. Q. Li, L. W. Mou, J. Y. Zhang, Y. H. Zhang, and L. W. Fan, "Enhanced pool boiling heat transfer during quenching of water on superhydrophilic porous surfaces: effects of the surface wickability," International Communications in Heat and Mass Transfer, vol. 125, pp. 494-505, 2018.

[5] F. Yokomatsu, W. Fogaça, S. Mori, and M. Tanaka, "On the quenching of stainless steel rods with a honeycomb porous 
plate on a nanoparticle deposited surface in saturated water," International Journal of Heat and Mass Transfer, vol. 127, pp. 507-514, 2018.

[6] R. Z. Xu, C. Y. Jin, T. Yu, and Y. C. Liu, "On quenching for some parabolic problems with combined power-type nonlinearities," Nonlinear Analysis: Real World Applications, vol. 13, no. 1, pp. 333-339, 2012.

[7] S. M. Zhou, C. L. Mu, Q. L. Du, and R. Zeng, "Quenching for a reaction-diffusion equation with nonlinear memory," Communications in Nonlinear Science Numerical Simulation, vol. 17, no. 2, pp. 754-763, 2012.

[8] H. Pan and R. Xing, "Fringing field can prevent infinite time quenching," Nonlinear Analysis: Real World Applications, vol. 30, pp. 126-142, 2016.

[9] J. Zhou, "Quenching for a parabolic equation with variable coefficient modeling MEMS technology," Applied Mathematics and Computation, vol. 314, pp. 7-11, 2017.

[10] Q. Wang, "Quenching phenomenon for a parabolic MEMS equation," Chinese Annual of Mathematics Series B, vol. 39, no. 1, pp. 129-144, 2018.

[11] S. M. Zhou and C. L. Mu, "Quenching for a reaction-diffusion system with coupled inner singular absorption terms," Boundary Value Problems, vol. 2010, no. 1, Article ID 797182, p. 15, 2010.

[12] B. Selcuk, "Quenching behavior of a semilinear reactiondiffusion system with singularboundary condition," Turkish Journal of Mathematics, vol. 40, pp. 166-180, 2016.

[13] I. D. Bonis and A. Muntean, "Existence of weak solutions to a nonlinear reaction-diffusion system with singular sources," Electronic Journal of Differential Equations, vol. 2017, no. 202, pp. 1-16, 2017.

[14] B. Selcuk and N. Ozalp, "The quenching behavior of a semilinear heat equation with a singular boundary outflux," Quarterly of Applied Mathematics, vol. 72, no. 4, pp. 747-752, 2017.

[15] Y. Ge, Z. Cai, and Q. Sheng, "A compact adaptive approach for degenerate singular reaction-diffusion equations," Numerical Methods in Partial Differential Equations, vol. 34, no. 4, pp. 1166-1187, 2018.

[16] M. A. Beauregard, "Numerical approximations to a fractional Kawarada quenching problem," Applied Mathematics and Computation, vol. 349, pp. 14-22, 2019.

[17] Q. Zhou, Y. Y. Nie, X. Zhou, and W. Guo, "Quenching of a semilinear diffusion equation with convection and reaction," Electronic Journal of Differential Equations, vol. 2015, no. 208, pp. 1-7, 2015.

[18] X. Zhu and R. Hong, "High-order compact difference scheme of 1D nonlinear degenerate convection-reaction-diffusion equation with adaptive algorithm," Numerical Heat Transfer, Part B: Fundamentals, vol. 75, no. 1, pp. 43-66, 2019.

[19] M. A. Beauregard and Q. Sheng, "A semi-adaptive compact splitting method for the numerical solution of 2-dimensional quenching problems," Applied Mathematics and Computation, vol. 218, no. 22, pp. 11240-11254, 2012.

[20] M. A. Beauregard and Q. Sheng, "Solving degenerate quenching-combustion equations by an adaptive splitting method on evolving grids," Computers and Structures, vol. 122, pp. 33-43, 2013.

[21] J. L. Padgett and Q. Sheng, "Numerical solution of degenerate stochastic Kawarada equations via a semi-discretized approach," Applied Mathematics and Computation, vol. 325, pp. 210-226, 2018.
[22] X. Zhu and Y. Ge, "Adaptive ADI numerical analysis of 2D quenching-type reaction: diffusion equation with convection term," Mathematical Problems in Engineering, vol. 2020, Article ID 8161804, 19 pages, 2020.

[23] S. H. Kwang, J. P. Rae, B. K. Sang, and D. K. Hee, "A highaccuracy finite difference scheme for solving reactionconvection-diffusion problems with a small diffusivity," Advances in Applied Mathematics and Mechanics, vol. 6, no. 5, pp. 637-662, 2014.

[24] L. Li, Z. Jiang, and Z. Yin, "Fourth-order compact finite difference method for solving two-dimensional convection-diffusion equation," Advances in Difference Equations, vol. 234, 24 pages, 2018.

[25] L. Wu and S. Zhai, "A new high order ADI numerical difference formula for time-fractional convection-diffusion equation," Applied Mathematics and Computation, vol. 7, pp. 110, 2019.

[26] W. Xue and Y. Ge, High-order compact difference schemes and multigrid algorithms on non-uniform grids for the twodimensional incompressible vorticity-stream function NavierStokes equations, Ningxia University, Yinchuan, 2014.

[27] X. Huang and Y. Ge, A high-order compact ADI method on non-uniform grid for unsteady convection-diffusion equations, Ningxia University, Yinchuan, 2013. 